\title{
Genetic Mapping of Brain Plasticity Across Development in Williams Syndrome: ERP Markers of Face and Language Processing
}

\author{
D. L. Mills \\ L. Dai \\ I. Fishman \\ A. Yam \\ L. G. Appelbaum \\ M. St. George \\ A. Galaburda \\ U. Bellugi \\ J. R. Korenberg
}

\section{QUERY SHEET}

This page lists questions we have about your paper. The numbers displayed at left can be found in the text of the paper for reference. In addition, please review your paper as a whole for correctness.

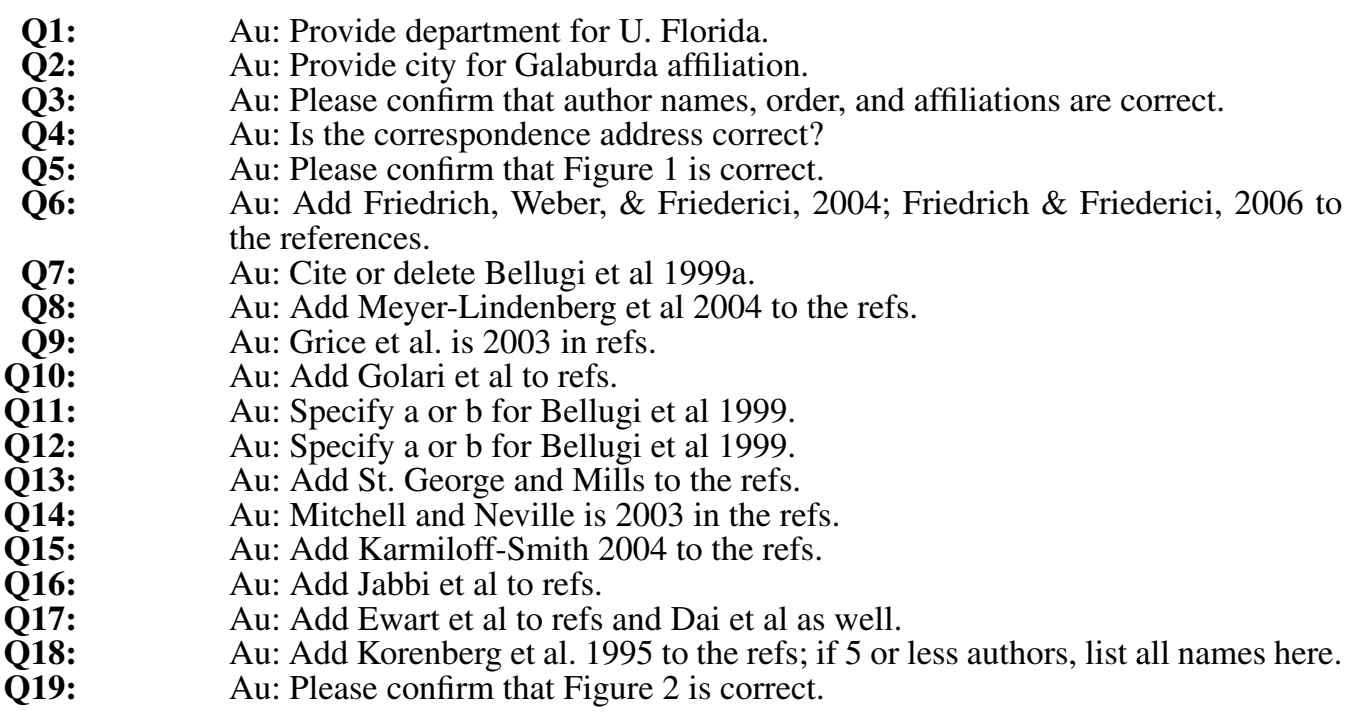




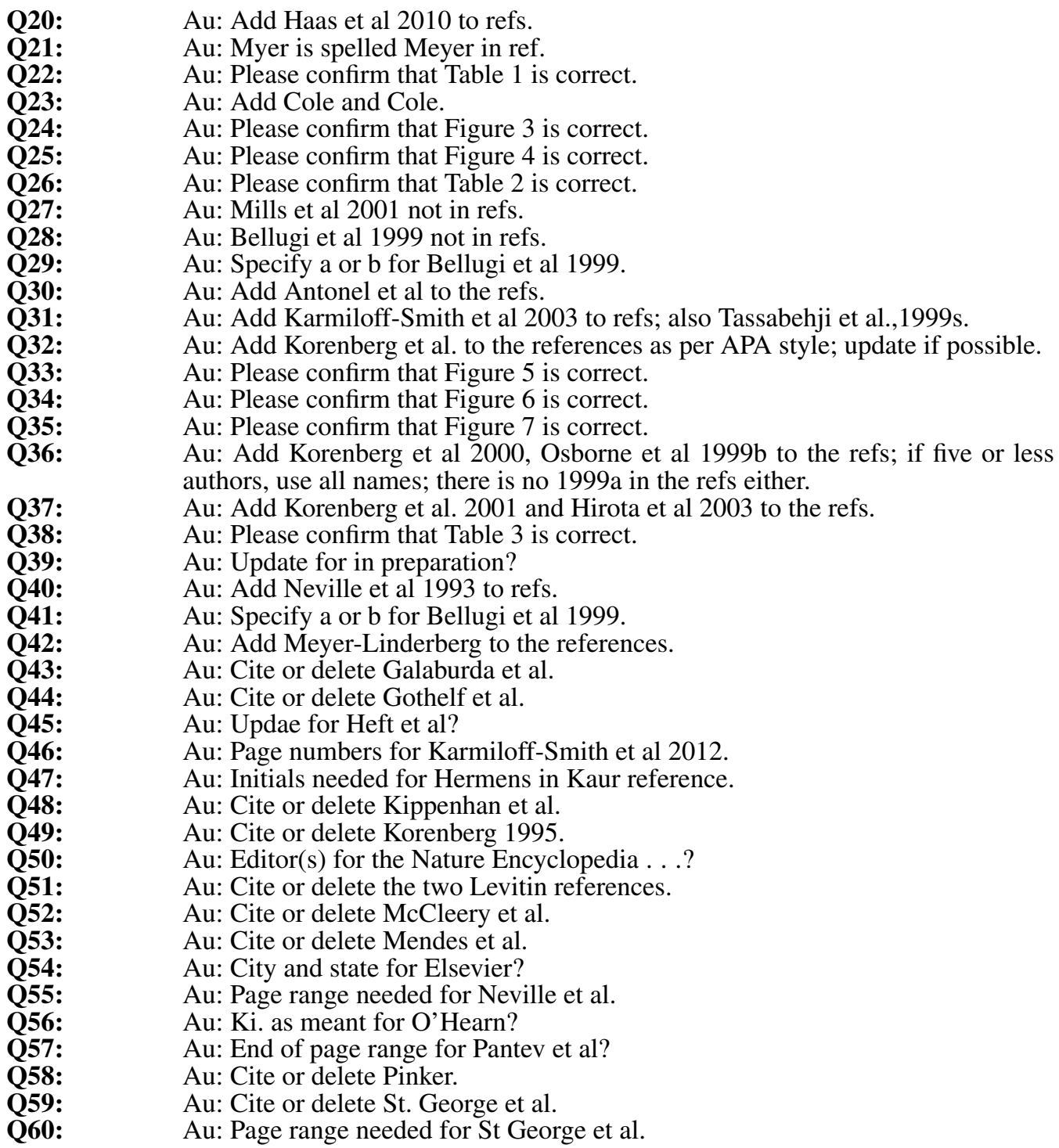

\section{TABLE OF CONTENTS LISTING}

The table of contents for the journal will list your paper exactly as it appears below:

Genetic Mapping of Brain Plasticity Across Development in Williams Syndrome: ERP Markers of Face and Language Processing

D. L. Mills, L. Dai, I. Fishman, A. Yam, L. G. Appelbaum, M. St. George, A. Galaburda, U. Bellugi, and J. R. Korenberg 


\title{
Genetic Mapping of Brain Plasticity Across Development in Williams Syndrome: ERP Markers of Face and Language Processing
}

\author{
D. L. Mills \\ School of Psychology, Bangor University, Bangor, United Kingdom
}

L. Dai

The Brain Institute, Department of Pediatrics, University of Utah, Salt Lake City, Utah

I. Fishman

Department of Psychology, San Diego State University, San Diego, California

A. Yam

Laboratory for Cognitive Neuroscience, The Salk Institute for Biological Studies,

La Jolla, California, and

University of Florida, Gainesville, Florida

L. G. Appelbaum

Department of Psychiatry and Behavioral Sciences, Duke University, Durham, North Carolina

M. St. George

Department of Behavioral Sciences, San Diego City College, San Diego, California

\section{A. Galaburda}

Department of Neurology, Beth Israel Deaconess Medical Center,

Harvard Medical School, Massachusetts

U. Bellugi

Laboratory for Cognitive Neuroscience, The Salk Institute for Biological Studies,

La Jolla, California

This research was supported by the National Institute of Child Health and Human Development (NICHD) grant (P01 HD033113) awarded to U.B., D.M., J.K., and A.R.

Correspondence should be addressed to D. L. Mills, ESRC Centre for Research on Bilingualism, School of Psychology, Adeilad Brigantia, Penrallt Road, Bangor LL57 2AS, UK. E-mail: d.1.mills@ bangor.ac.uk 


\author{
J. R. Korenberg \\ The Brain Institute, Department of Pediatrics, University of Utah, Salt Lake City, Utah
}

In Williams Syndrome (WS), a known genetic deletion results in atypical brain function with strengths in face and language processing. We examined how genetic influences on brain activity change with development. In three studies, event-related potentials (ERPs) from large samples of children, adolescents, and adults with the full genetic deletion for WS were compared to typically developing controls, and two adults with partial deletions for WS. Studies 1 and 2 identified ERP markers of brain plasticity in WS across development. Study 3 suggested that in adults with partial deletions for WS, specific genes may be differentially implicated in face and language processing.

Establishing links across genes, brain, and behavior is a central issue in cognitive neuroscience research. Neurodevlopmental studies of typical and atypical populations have the potential to elucidate how these factors interact to shape the specialization of the brain for different cognitive functions. Yet very little research on how genes affect the neuroplasticity of the developing brain has been published. In three studies, we employed a combined genetic, electrophysiological, and developmental approach to examine markers of brain plasticity in a rare genetic disorder, Williams Syndrome (WS).

In WS, the deletion of approximately 25-28 genes on chromosome 7q11.23 (Figure 1) results in an unusual pattern of cognitive and behavioral strengths and deficits, and specific alterations in brain morphology (Bellugi, Lichtenberger, Jones, Lai, \& St. George, 2000; Bellugi \& St. George, 2001). Individuals with WS typically show a strong drive for social interaction and demonstrate marked strengths in face and language processing. These characteristics are particularly striking

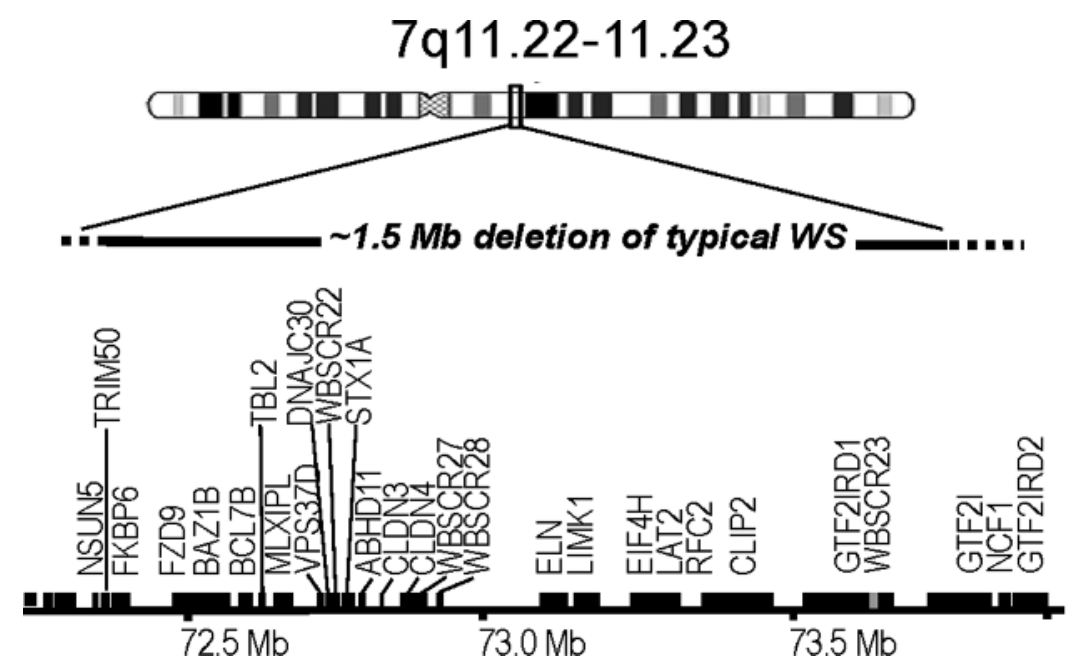

FIGURE $1 \sim 1.5 \mathrm{Mb}$ genetic deletion on Chromosome 7q11.23 in typical Williams Syndrome. 
in the face of global intellectual disability and severe deficits in spatial and numerical abilities (Bellugi et al., 2000).

To date, studies of brain structure and function in WS have only been conducted with adults. The extent to which relations between genes, brain, and behavior in WS may emerge as a function of development is unknown. The present study examines developmental trajectories of brain activity linked to face and language processing in large samples of individuals with the typical deletion for WS. This approach allows us to examine emerging neurocognitive profiles related to the deletion of all of the WS region genes.

An important tool in studying brain function in a variety of neurodevelopmental disorders has been the identification of biomarkers. For example, electroencephalography (EEG) and eventrelated potential (ERP) biomarkers have been used to predict later outcomes for infants or young children at risk for neurodevelopmental or psychiatric disorders such as: specific language impairment (Friedrich, Weber, \& Friederici, 2004; Friedrich \& Friederici, 2006), Dyslexia (Molfese, 2000), attention deficit hyperactivity disorder (Klein, Wendling, Huettner, Ruder, \& Peper, 2006; Loo \& Makeig, 2012), and schizophrenia (Kaur et al. 2012). Electrophysiological markers may be particularly useful to identify children at risk in disorders where there is no genetic test as in autism or language impairment. In the specific case of WS, biomarkers of brain function may have a variety of additional uses including: (a) examining individual differences and group variability in neurocognitive profiles, (b) establishing if a particular individual falls within the range for WS or controls, (c) correlating markers of brain activity and performance on behavioral measures, (d) establishing developmental trajectories of brain activity, and (e) to examine the role of individual genes on biomarkers of brain activity for individuals who have partial deletions in the WS range.

One goal of the present research was to determine if atypical patterns of brain activity characteristic of face and language processing, areas of cognitive strength observed in adults with WS, could be used as ERP markers of brain function in children and adolescents with full deletions for WS. A secondary goal was to examine the role of individual genes in producing the characteristic WS profile, by examining variability in these ERP markers in two individuals with partial genetic deletions. By studying individuals with smaller deletions in this region, we may begin to elucidate the role of individual genes.

WS is associated with mild to moderate reductions in intellectual abilities (Martens, Wilson, $\&$ Reutens, 2008). Of particular interest is that the intellectual profile in WS varies across cognitive domains. The typical WS profile consists of deficits in spatial and numerical abilities, with relative proficiencies in face and language abilities that are higher than predicted by IQ (Bellugi et al., 2000). Note that relative proficiencies are not the same as absolute proficiencies, and do not denote normal performance. Cognitive strengths in face and language processing are relative to overall intellectual performance and cognitive deficits in other domains. Regional abnormalities in brain morphology appear consistent with relative strengths and weaknesses in the WS cognitive profile. One explanation for the WS neurocognitive profile suggests a dichotomy in normal and abnormal brain function along the ventral and dorsal stream pathways. The dorsal/ventral dichotomy hypothesis purports that in WS dorsal stream processes are impaired, whereas ventral stream processes are spared (Landau, Hoffman, \& Kurz, 2006; Meyer-Lindenberg, Mervis, \& Berman, 2006). Support for this hypothesis comes from several lines of evidence including behavioral studies of visuospatial deficits associated with dorsal stream processes (Atkinson et al., 2006; Bellugi et al., 1999a; Landau et al., 2006; Wang, Doherty, Rourke \& Bellugi, 1995), and magnetic resonance imaging (MRI) studies showing abnormalities in dorsal regions (Eckert, 
et al., 2005; Meyer-Lindenberg, et al., 2004; Reiss et al., 2000). Additionally, complementary patterns of cell size and cell packing density have been found in visual dorsal (Galaburda \& Bellugi, 2000) and auditory ventral (Holinger et al., 2005) areas.

Links between atypical brain structure and cognitive profiles are compelling but do not necessarily provide information about the functional specialization of either pathway. Studies of brain function using ERPs were the first to suggest that putatively spared ventral stream processes such as face (Korenberg, Bellugi, Salandanan, Mills, \& Reiss, 2003; Grice et al. 2005; Mills et al., 2000) and language processing (Bellugi, Lichtenberger, Mills, Galaburda, \& Kore, 1999b; Neville, Holcomb, \& Mills, 1989), are abnormally organized in WS. More recent studies have provided converging evidence using a variety of methods that ventral stream activity is neither normal nor "spared" in WS (Fishman, Yam, Bellugi, Lincoln, \& Mills, 2011a; Golari et al., 2010; Haas et al., 2009; Leonard, Annaz, Karmiloff-Smith, \& Johnson, 2011; O’Hearn et al., 2011).

For example, in an ERP face recognition task, adults with WS showed marked differences from typically developing (TD) controls in patterns of brain activity for recognition of upright versus inverted faces (Mills et al., 2000). Although WS and controls showed similar behavioral inversion effects, the two groups showed marked differences in the organization of brain activity mediating these effects. TD controls showed different ERP effects for recognition of upright (N320) and inverted (P600) faces. In contrast to controls, adults with WS showed the same N320 effect for both upright and inverted faces. That is, adults with WS processed both upright and inverted faces as if they were upright.

ERP studies of auditory sentence processing in adults with WS also demonstrate atypical organization of brain activity linked to different aspects of language (Bellugi et al., 1999; Neville et al., 1989). Individuals with WS showed an abnormally large N400 semantic integration effect to anomalous sentence endings relative to TD controls and adults with autism (Fishman et al., 2011a). Moreover the amplitude of the N400 effect was linked to sociability ratings in WS but not controls (Fishman, Yam, Bellugi, \& Mills, 2011b). Additionally, in spite of relative behavioral proficiencies in tests of grammatical processing, adults with WS do not show typical ERP left hemisphere asymmetries or differences between auditory words conveying semantic content (open class) versus grammatical function (closed class) words (Bellugi et al., 1999; Mills, 1998; Neville, 1993; St. George \& Mills, 2000).

Atypical brain function associated with cognitive proficiencies suggests that brain plasticity, rather than spared versus impaired functioning, may account for the unusual WS neurocognitive profile. There are well-established differences in the vulnerability, plasticity, and time-course of development for dorsal compared to ventral stream processes (see Mitchell \& Neville, 2002). Abnormalities in dorsal stream processing have been reported in a variety of different disorders including autism, language impairment, and schizophrenia (Pellicano, Gibson, Maybery, Durkin, \& Badcock, 2005; Ullman \& Pierpont, 2005). Thus, deficits in visuospatial skills are not unusual in clinical populations nor are they specific to WS. It is the relative and marked cognitive proficiencies in the presence of lower IQ, smaller brains, and other cognitive and physical deficits that characterize the unusual profile in WS. Therefore, studying atypical brain activity associated with cognitive strengths is probably the most likely approach to yield patterns of brain plasticity unique to WS.

Here ERPs are used to investigate patterns of brain activity that may reflect atypical/compensatory processing within the ventral stream in WS. Of particular interest were ERP components associated with marked group differences in brain activity between WS and 
controls linked to face (Mills et al., 2000) and language (Fishman et al., 2011a; Neville et al., 1989) processing. These ERP studies also showed that adults with WS reliably displayed abnormal morphology of ERP components to both faces and auditory words within the first $200 \mathrm{msec}$ linked to sensory and attentional processes (Bellugi et al., 1999b). We reasoned that the probability of detecting ERP markers of abnormal processing would be more likely in these early components than in later components, which displayed more variability. To characterize the ERP phenotypes or markers of atypical brain function characteristic of face and language processing in WS, composite scores were calculated from measurements of early components, that is, within $200 \mathrm{msec}$ after stimulus onset, observed for face and language processing paradigms. The term "ERP marker" refers to the functional significance of the set of ERP components identified for each task. Thus, these were not meant as ERP markers for WS per se, but markers of atypical patterns of brain activity linked to face and language processing characteristic of individuals with WS. The phrase "ERP markers of brain plasticity" was adopted to describe the patterns of abnormal brain activity linked to these two cognitive strengths in WS.

To our knowledge, the only published studies of brain function in WS have been conducted with adults. Therefore, it is not clear how patterns of brain activity from children and adolescents with WS differ from that of controls or adults with WS. Karmiloff-Smith and colleagues point out that both face and language abilities in adults with WS stem from deviant developmental trajectories (Karmiloff-Smith, 2004; Karmiloff-Smith \& Thomas, 2003). For example, KarmiloffSmith et al. (2004) examined developmental trajectories for different aspects of face processing in WS from childhood through adolescence. Developmental changes in configural processing of faces and the inversion effect showed both deviance and delay in WS. It stands to reason that brain activity linked to these cognitive strengths in adults, could differ from children with WS who show deviance and delay. Karmiloff-Smith also argues that the process of development must be considered to fully understand complex and indirect relations across levels in mapping genes to phenotypes (Karmiloff-Smith, Scerif, \& Thomas, 2001). Additionally, a recent paper linking structure and function in the anterior insula with the WS personality (Jabbi et al., 2012) calls for studies tracking the trajectory of neural function as a measure of neuroplasticity in the developing WS brain to help explain gene environment interactions. Therefore, it is important to establish how WS neurocognitive profiles develop across the lifespan. To this end, we chart developmental trajectories in ERP markers for face and language processing across development in WS and typically developing (TD) controls.

In the first two studies, ERPs to faces (Study 1) and auditory language (Study 2) were recorded from large numbers of participants with WS with the typical deletion and age and gender matched TD controls between the ages of 8 and 51 years. To illustrate developmental trajectories and chart individual variability within the WS and TD populations, the composite scores were plotted as scattergrams for each age group. The composite scores were based on sets of ERP amplitudes that showed marked differences between WS and TD adults for face and language processing as described above. The specific components used in calculating these composite scores, and the direction of expected amplitude differences are described in the short introductions to each study. Discriminant analyses were used to evaluate the effectiveness of the composite scores as markers of brain plasticity in WS across development. We tested the contrasting hypotheses that the neural phenotypes for face and language processing in WS are apparent early in development, or emerge with increasing age. Developmental changes in ERP markers for each paradigm were investigated by examining age-related trends using regression analyses, and the effectiveness of 
the marker to discriminate between groups separately in children, adolescents, young adults, and 180 middle-aged adults. The third study examined the utility of the ERP markers in generating and testing hypotheses regarding the effect of genetic variability on brain plasticity. This study tested the effect of genetic variability on the ERP phenotypes by comparing individual variability within the WS and TD groups to two WS individuals with smaller deletions. Z-scores were calculated to determine if each individual's composite scores fell within the range for WS or TD controls. These results were discussed in light of previous studies examining the role of genes at different ends of the deleted regions in WS, GTF2IRD1, and FKBP6, on face and language processing, respectively.

\section{GENERAL METHODS}

Methods in common to all three studies are described first.

\section{Participants}

Individuals with WS were recruited through a large multicenter collaborative research project. ${ }^{1}$ Genetic analyses of the WS deleted region in the WS participants employed multicolor fluorescence in situ hybridization (FISH) using probes for DNA sequences that flank the common form of the 7q11.23 deletion, combined with probes for elastin, a gene found within the region (Ewart et al. 1993; Korenberg et al. 1995). To refine the deletion breakpoints, subsequent FISH studies used multiple subsets of BAC (bacterial artificial chromosome), PAC (cloning vector), and cosmid probes that spanned the entire common WS deleted and flanking duplicated regions for the 75 WS participants (Dai et al., 2009). In addition, a medical geneticist (author JRK) evaluated all clinical data for WS features. All diagnoses were consistent with the WS Diagnostic Score Sheet, developed by the Medical Advisory Board of the Williams Syndrome Association. Participants were excluded if they had other neurological or neuropsychiatric conditions not typically associated with WS. Overall level of intellectual functioning for the participants with WS was assessed through the multicenter using the Wechsler Scale of Intelligence for Adults or Children (WAIS-R or WAIS-III, WISC-R or WISC-III, respectively) depending on their age and date of testing. Additionally the WS participants were tested on the Benton Test for Face Recognition (BFRT, Benton, Hamsher, Varney, \& Spreen, 1983).

Typically developing controls were recruited through advertisements in local newspapers, and fliers posted in local areas and schools. All participants were monolingual speakers of English and had normal or corrected to normal vision and hearing. Participants were paid to compensate for their time and travel costs. All protocols were approved by the University Institutional Review Board. Participants or their parents or guardians signed informed consents.

\footnotetext{
${ }^{1}$ The multicenter was supported by the National Institute of Child Health and Human Development (NICHD) grant (P01 HD033113) awarded to U.B., D.M., J.K., and A.R.
} 


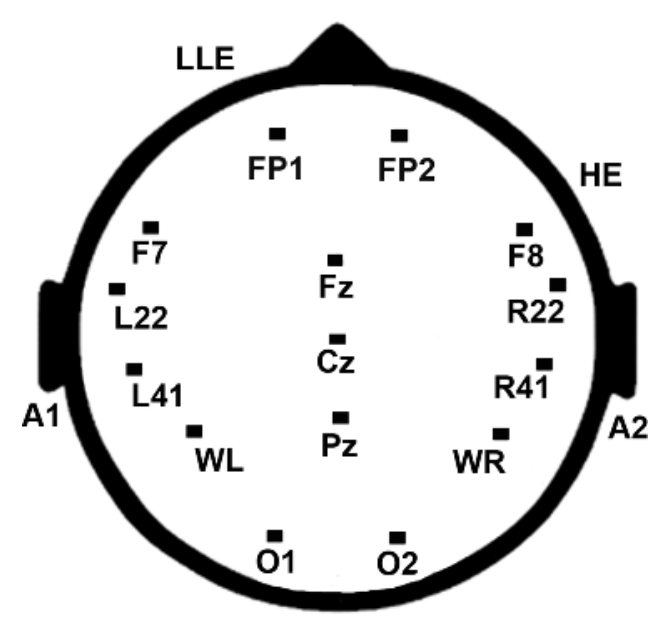

FIGURE 2 Illustration of electrode placements for standard and nonstandard recording sites.

\section{Electrophysiological Recording}

The electroencephalogram (EEG) was recorded using tin electrodes from 14 sites affixed to a cap (Electro-cap International) and two EOG sites (Figure 2). Eight of the sites were from standard 10/20 placements over left and right fronto-central, (Fp1/Fp2), frontal (F7/F8), occipital $(\mathrm{O} 1 / \mathrm{O} 2)$, and midline positions, $(\mathrm{Cz}$, and $\mathrm{Pz})$, and six were nonstandard sites designed to be located over areas along the perisylvian fissure including anterior temporal (L/R22, which was situated one-half the distance between F7/F8 and T3/T4), temporal (L/R41, which was situated $33 \%$ of the distance from $\mathrm{T} 3 / \mathrm{T} 4$ to $\mathrm{C} 3 / \mathrm{C} 4$ ), left and right temporo-parietal (WL/WR, which was $50 \%$ of the distance between $\mathrm{T} 3 / \mathrm{T} 4$ and P3/P4). Additionally, the electrooculogram was recorded from over (Fp1) and under the left eye (Le) to monitor blinks and vertical eye movements and from the right outer canthus $(\mathrm{He})$ to monitor horizontal eye movements. Impedances were maintained below $5 \mathrm{KOhms}$. All electrodes were referenced to the average of the left and right mastoids. The EEG was amplified at a gain of 10,000 using SA Instrument amplifiers (input impedances of 1,000 Meg Ohms per channel), recorded at $250 \mathrm{~Hz}$, and filtered with a bandpass of .01 to $100 \mathrm{~Hz}$. The averaged ERPs were also digitally filtered offline with a $60 \mathrm{~Hz}$ low-pass filter. A wide band-pass is recommended to minimally distort the ERPs (Luck, 2005). Electrical noise was not observed in the individual averages. This procedure was used to be consistent with previous research using these paradigms (Fishman et al., 2011a,b; Mills et al. 2000).

\section{ERP Data Reduction and Analysis}

Artifact rejection was conducted offline using a computer program in the data analysis ERPSS software (Event Related Potential Software System, J. S. Hansen, Event-Related Potential Laboratory, University of California San Diego, La Jolla, CA, 1993). As expected, amplitude fluctuations associated with blinks and other movement artifacts varied across age thereby rendering 
a single artifact rejection threshold unfitting. Criteria for setting thresholds for rejection of trials containing eye blinks, horizontal eye movement, muscle movement, or amplifier blocking were set for each participant individually based on visual inspection of the data on a trial-by-trial basis (as recommended by Luck, 2005). Across all groups and both paradigms, an average of 60\% (range 40 to $100 \%$ ) of the trials were artifact-free and retained for analysis.

\section{STUDY 1. DEVELOPMENTAL CHANGES IN ERP MARKERS OF FACE PROCESSING}

The present study used the same stimuli and design as Mills et al. (2000). In that study, adults with WS showed marked differences from controls in response to both perception and recognition of unfamiliar upright and inverted faces. Of particular interest here, the WS group reliably displayed abnormal patterns of activity within the first $200 \mathrm{msec}$ after stimulus onset (N1 and N2). WS participants showed an abnormally small negative peak at $100 \mathrm{msec}(\mathrm{N} 1)$ relative to an abnormally large negative component at $200 \mathrm{msec}$ (N2). This pattern was observed for all WS participants. In contrast, most TD controls showed the opposite pattern (i.e., larger N1 than N2). A smaller subset of controls showed equal amplitude N1 and N2 responses.

The authors hypothesized that the small N1 in WS indexed abnormal visual sensory processing and the large N2 indexed increased attention to faces. Functional magnetic resonance imaging (fMRI) evidence on face/gaze processing in WS showed decreased activation to faces in the occipital region linked to primary visual processes, and increased activation in the right prefrontal regions and the anterior cingulate, which is thought to modulate attention (Mobbs et al., 2004). The visual N1 amplitude has been linked to a variety of early sensory and attentional processes as well as orienting and discrimination (for a review see Pratt, 2012). Although the of the N1 has not been studied systematically in WS, reduced amplitude of the visual N1 has been proposed to serve as an endophenotype for susceptibility to psychiatric disorders (Smit, Posthuma, Boomsa, \& De Geus, 2007). The amplitude of the N2 has been shown to correlate with reduced amygdala activity to fearful expressions (Haas et al., 2009). Reduced amygdala activity to fearful expressions has been linked to individual differences in the WS social phenotype (Haas et al, 2010; Myer-Lindenberg et al., 2005).

The reliability of the $\mathrm{N} 2 / \mathrm{N} 1$ pattern in WS along with the functional significance of the $\mathrm{N} 1$ and $\mathrm{N} 2$ components to characterize both abnormal visual processing and the social phenotype, make the composite a good candidate for an ERP marker for face processing in WS. We predicted that the characteristic ERP pattern observed for WS (i.e., a small N1 and relatively larger N2), would serve as an ERP marker for abnormal face processing in WS. We tested the hypothesis that this relation would be observed at each age group across development.

\section{Method: Face Processing}

Participants. The adult and child participants ( 8 to 51 years of age) included 140 typically developing (TD) controls and 74 individuals with WS. The number of participants at each age group for each paradigm and group is shown in Table 1. Approximately half of the participants at each age group for each population were female. The participants consisted of all WS recruited by the multicenter with useable ERP data on this paradigm. Data for the TD group included all 
TABLE 1

Participants in Face Processing and Auditory Language Paradigms Including Typically Developing (TD) and Williams Syndrome (WS) Children and Adults

\begin{tabular}{lcccc}
\hline & \multicolumn{2}{c}{ Face Processing } & \multicolumn{2}{c}{ Auditory Language } \\
\hline Age in years & $T D$ & WS & $T D$ & WS \\
$8-12$ & 34 & 11 & 42 & 15 \\
$13-15$ & 36 & 11 & 35 & 16 \\
$17-29$ & 47 & 29 & 45 & 27 \\
$30-51$ & 23 & 24 & 13 & 17 \\
Total: & 140 & 75 & 135 & 75 \\
\hline
\end{tabular}

participants tested on this paradigm who were in the same age ranges as the WS participants. That is, groups were not specifically matched on any variable other than age range.

For illustrative purposes (Figures 3 and 4), and for specific analyses designed to investigate group differences at specific points in development, the sample was divided into four age groups.

\section{FACE PROCESSING}

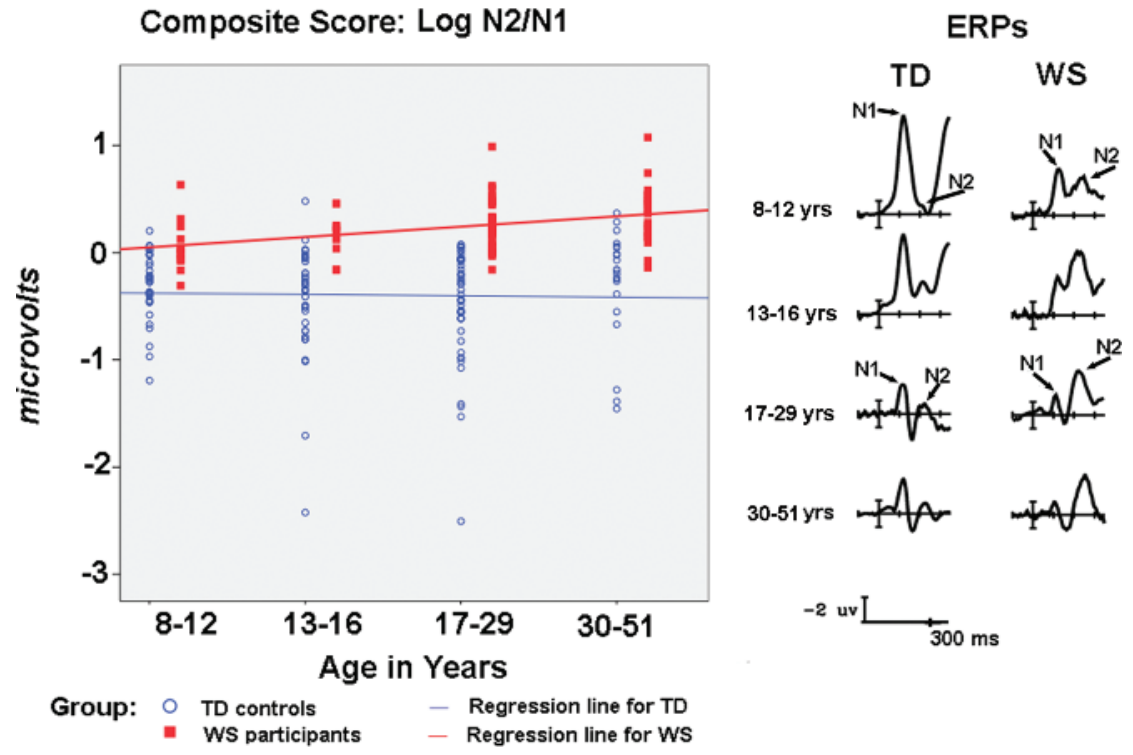

FIGURE 3 Event-related potentials (ERPs) and composite scores for face processing. The right side of the figure depicts ERPs to faces in the first $300 \mathrm{msec}$, showing the relatively larger N2 than N1 responses in Williams Syndrome (WS) and the opposite pattern in typically developing (TD) controls across development. The left side shows scattergrams for the composite scores (Log N2/N1) of each participant for each age group, showing that group differences are evident from childhood through middle age. (color figure available online) 


\section{AUDITORY LANGUAGE}

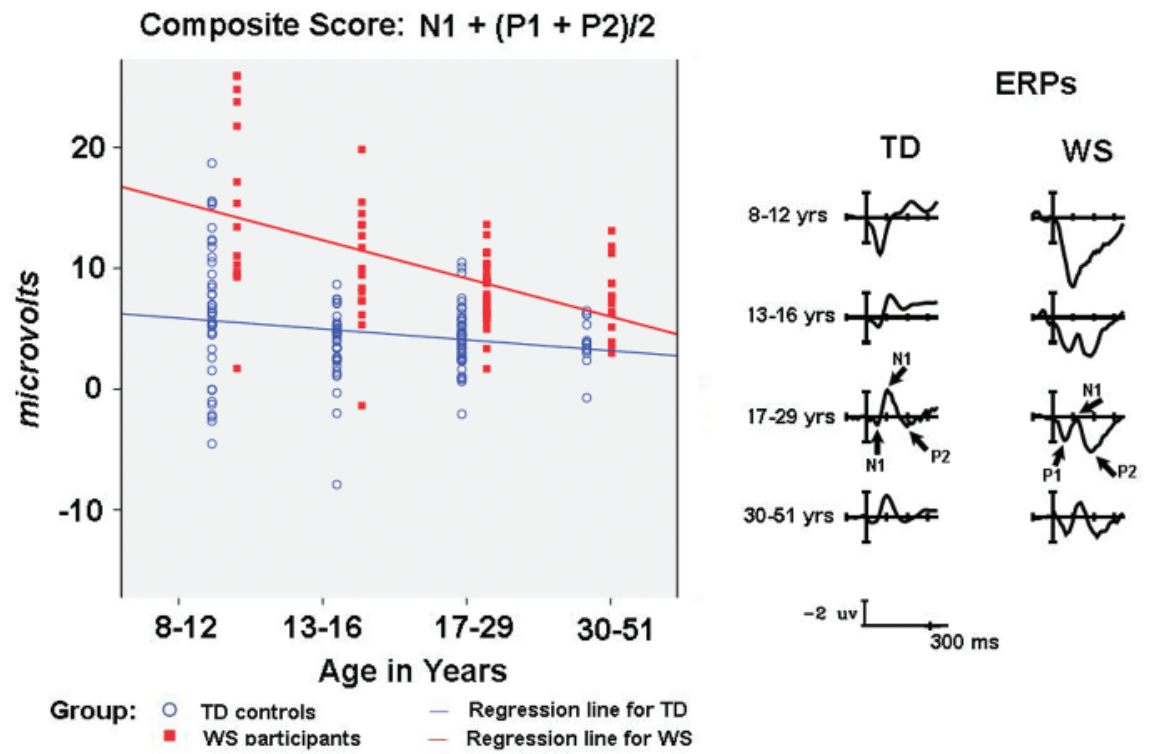

FIGURE 4 Event-related potentials (ERPs) and composite scores for auditory language processing. The right side of the figure shows ERPs to auditory language stimuli in the first $300 \mathrm{msec}$, showing the large P1 and P2 and small N1 pattern observed in children and adults with Williams Syndrome (WS). In contrast typically developing (TD) children and adults show a large $\mathrm{N} 1$ and reduced amplitude $\mathrm{P} 1$ and $\mathrm{P} 2$ responses. The left side illustrates individual variability in composite scores (N1 + mean of P1and P2) for WS and TD children and adults, showing that the WS pattern becomes more similar to TD controls with increasing age. (color figure available online)

This approach was adopted to test the hypothesis that the WS phenotype would differ from TD 280 controls at some but not all age groups. Age groups were chosen to characterize widely accepted divisions or stages of development (of Cole \& Cole, 2001), but were also constrained by the sample of WS participants. Overall, the groups consisted of children (8-12 years), adolescents (13-15), young adults (17-29), and middle adulthood (30-51). For all other analyses age was entered as a continuous variable. The terms "age group" and "age" are used here to signify when age was used as a grouping or continuous variable respectively.

Standardized measures were only available for the WS group. Measures for IQs: Verbal IQ = $70, S D=10$; Performance IQ $=65, S D=10$; Full Scale IQ $=66, S D=10$. Consistent with the literature IQs for WS participants were below average (Martens et al., 2008). The WS participants scored in the normal range on the BFRT. The mean raw scores and standard errors by age group in years were: $8-12=18.6$, se $=1.2 ; 13-15=21.7$, se $=1.3,17-29=21.17$, se $=.542,30-51$ $=20.62$, se $=.551$. The BFRT is often given as a measure of face recognition abilities in children 
and atypical populations. The performance on the BFRT displayed here is typical of adults and children with WS (Annaz, Karmiloff-Smith, Johnson, \& Thomas, 2009).

Stimuli and procedure. Participants viewed digitized black and white photographic pairs of upright or inverted faces (half female) on a computer screen (Mills et al., 2000). To control for hairstyle, all models of the same gender wore the same wig. There were 38 pairs of faces per condition. Trials consisted of the sequential presentation of two faces. ERPs analyzed here are to the second, target, face in the upright orientation.

On half of the trials, the identity of the two faces in the pair matched. That is, they were different photographs of the same person. On the other half, the photographs were of different people with similar face shapes. Participants were asked to press a button indicating whether the second face (target) matched or did not match the first (prime). Several of the children with WS had trouble understanding the button press. They would look down at the response box, eliciting eye artifact on each trial. Therefore, they were asked verbally if the faces matched or not, and an experimenter pressed the button for them. Because of this difference in the instruction and behavioral performance across groups, behavioral results are not included in the following analysis. In general, adults with WS have been found to be slower and approximately $10 \%$ less accurate than TD controls on comparable tasks (Mills et al., 2000).

Measurement of ERP components. Peak amplitudes ${ }^{2}$ were quantified by computer with reference to the $100 \mathrm{msec}$ pre-stimulus baseline for the local maximum negative or positive point in two specified time windows, 75 to $175 \mathrm{msec}$ (N1) and 150 to $275 \mathrm{msec}$ (N2), according to procedures described in Mills et al. (2000). Time windows for children were 75-175 (N1) and 175-300 (N2). ERP components were measured at and averaged across eight electrode sites F7/8, $\mathrm{L} / \mathrm{R} 22, \mathrm{~L} / \mathrm{R} 41, \mathrm{WL} / \mathrm{R}$, which were previously found to reflect a large $\mathrm{N} 2$ to face processing in WS (Mills et al., 2000). Visual inspection of all individual averages, for each condition, and each electrode site, showed that these time windows accurately measured the components for all age groups.

Composite score for face processing. Consistent with previous findings for adults, the characteristic pattern for TD controls was a larger N1 than N2. In contrast, most participants with WS showed a larger $\mathrm{N} 2$ than $\mathrm{N} 1$. To characterize this relation of the $\mathrm{N} 2$ to $\mathrm{N} 1$, a composite variable was calculated for each participant as a ratio of the $\mathrm{N} 2$ divided by the $\mathrm{N} 1$. Because children are known to have markedly larger ERP amplitudes than adults (Holcomb, Coffey, \& Neville, 1992), the scale needed to be adjusted to characterize the N2/N1 relation across development. Large differences in peak amplitudes can artificially lead to significant differences across age groups. Log ratios are often used to equate for marked amplitude differences. Thus, we calculated the $\log$ of the amplitude ratio of the $\mathrm{N} 2 / \mathrm{N} 1$ to allow for scaling of all age groups to comparable values. The use of a single composite score to characterize the relationship between specific components across electrode sites is advantageous to test for group differences while minimizing the number of significance tests.

\footnotetext{
${ }^{2}$ We are aware of potential methodological issues related to measurement of peak versus mean amplitude measures. The previous study analyzed both peak amplitudes and mean amplitudes over the same time window and reported the same patterns of statistical significance.
} 


\section{Results}

The ERP waveforms to upright faces for TD controls and participants with WS at each age group are shown on the right side of Figure 3. Marked differences in the N1 and N2 were observed between groups at each age group. Individual composite scores are illustrated in the scatter plots on the left side of Figure 3. A score of zero indicates that the N1 and N2 were of equal amplitudes. A positive score reflects the typical WS pattern with a larger N2 than N1. A negative score reflected the TD pattern with a larger N1 than N2.

Examination of individual composite scores across all ages showed that 67 out of 74 participants with WS (91\%) displayed the typical WS pattern (composite score $\geq 0.01$, with two displaying a score of 0.00$)$. For TD participants, 119 out of $140(85 \%)$ showed the TD pattern (composite score $\leq-0.01$ ).

Gender and IQ as potential covariates. To examine possible gender differences, a group $\mathrm{x}$ gender ANOVA was conducted with age as a covariate for the composite score. There were no significant main effects or interactions with gender for composite score, main effect of gender, $F(1,214)=.1055, p=.815$; gender $\mathrm{x}$ group, $F(1,214)=.689, p=.407$. Nor were there gender differences for the $\mathrm{N} 1$, main effect of gender $F(1,214)=.144, \mathrm{p}=.705$, gender $\mathrm{x}$ group, $F(1,214)$ $=.045, p=.833$; or the $\mathrm{N} 2$ separately, main effect of gender $F(1,214)=.574, p=.449$, gender $\mathrm{x}$ group $F(1,214)=.233, \mathrm{p}=.630$. Therefore gender was removed as a factor from the analyses.

IQ scores were not available for the TD controls. Therefore IQ could not be entered into the analyses as a covariate. Thus, we examined the relation between FSIQ and ERP components for the WS group. For WS participants, IQ was not correlated with any of the ERP component amplitudes, FISQ and $\log \mathrm{N} 2 / \mathrm{N} 1$ composite scores $r(60)=.025, p=.844$; N2 amplitudes, $r(60)$ $=-.133, p=.303$; and N1 amplitudes, $r(60)=-.047, p=.716$. Similar null effects were obtained for VIQ and PIQ separately, and the BFRT.

Group differences. To determine the effectiveness of the composite score as a predictor of group membership, a discriminant analysis (SPSS19) was conducted with the faces log $\mathrm{N} 2 / \mathrm{N} 1$ composite score as a single predictor. The discriminant analysis revealed that $88.3 \%$ of cases were correctly identified, $\chi 2(d f=1)=96.699, p=.0001$, Wilks' Lambda $=.636$, Eigenvalue $=.573$. For the TD group $95.7 \%$ were correctly identified. For the WS group $75 \%$ were correctly identified.

A second discriminant analysis was conducted using the N1 and N2 as simultaneous factors in comparison to the results from the composite score. The discriminant analysis using the $\mathrm{N} 1$ and $\mathrm{N} 2$ entered together correctly identified $81.8 \%, \chi 2(d f=2)=118.137, p=.0001$, Wilks' lambda $=.571$, Eigenvalue $=.750$. That is, this was slightly less predictive than the composite score.

Separate discriminant analyses for the N1 and N2 components alone were both significant ( $p=$ .0001), but were less successful than the composite score, and combined analysis. Under these separate discriminant analyses, the $\mathrm{N} 1$ showed $74.3 \%$ correct group assignment, and $\mathrm{N} 2$ showed $65 \%$ correct group assignment. Thus, the Log N2/N1 composite score was the best predictor of group membership.

For the 19 WS participants misclassified as TD, 10 were adults and 10 were female. Those misclassified tended to be younger (mean age $=20$ ) than those correctly identified (mean age $=$ 26 ), $t(72)=-2.201, p=.031$. There were no differences between groups in FSIQ (misclassified 
as $\mathrm{TD}=62$; correctly classified as WS $=67), t(61)=-1.276, p=.207$, or on the Benton Face Recognition Test, $t(71)=-1.027, p=.308$.

Group differences across age. To investigate whether the marker differed for each group across development, $t$-test comparisons for the composite scores at each age group were conducted. Significance levels are reported for equal variance not assumed when Levine's test for equal variance was significant. $T$-tests revealed that the composite score differed for WS and TD controls for all age groups: at $8-12$ years, $t(43)=-3.914, p \leq .001 ; 13-16$ years, $t(45)$ $=-6.223, p \leq .0001 ; 17-29$ years, $t(74)=-8.236, p \leq .0001 ; 30-51$ years, $t(45)=-5.766$, $p \leq .0001$.

To further examine whether there were group differences in developmental trajectories, a regression analysis was conducted using group, age, and age $\mathrm{x}$ group as factors, $\mathrm{R}^{2}=.37$, $F(3,211)=41.169, p<.0001$. Only the effect of group was significant, group $b=.519, t(211)$ $=3.64, p \leq .0001$; age $b=-.003, t(211)=.353, p=.72$; age $\mathrm{x}$ group $t(211)=.836, p=.40$. Neither group showed an effect of age. Thus, there were no significant age related trends in the composite scores. Note that in this analysis age was a continuous variable.

Individual variability. It is often assumed that individual variability is greater in atypical than typically developing groups. We therefore compared the range, standard deviations, and variance statistics between the TD and WS groups. The results suggest that the WS group displayed less variability on all of these measures than did the TD group (Table 2a), Levine's test for equal variance $F(3,210)=5.684, p=.001 .^{3}$

Summary of ERP markers of face processing. ERP studies of adults with WS show that normal or near normal accuracy in face recognition is associated with abnormal patterns of activation for early sensory and attentional processes during face processing in adults with WS (Mills et al., 2001). In the present study, with large numbers of participants across development, patterns of brain activity elicited in the face recognition task showed marked differences from TD controls. The discriminant analysis showed that $88.3 \%$ of cases were correctly identified as

TABLE 2

Descriptive Statistics Showing Variability for TD and WS Participants for Face Processing (2A) and Language Processing (2B)

\begin{tabular}{lcccr}
\hline Measure & Range statistic & Mean & Std. deviation & Variance \\
\hline & & 2A. Face Processing Composite: Log $N 2 / N 1$ & .227 \\
TD (135) & 2.99 & -.395 & .255 & .065 \\
WS (75) & 1.38 & .259 & .255 & 12.3 \\
& & & \\
TD (140) & 24.15 & -.226 & Language Processing Composite: $N 1+(P 1+P 2) / 2$ \\
WS (74) & 24.79 & 4.315 & 4.937 & 24.38 \\
\hline
\end{tabular}

\footnotetext{
${ }^{3} \mathrm{We}$ are aware that regression analysis assumes equal variance across groups. Unequal variance could underestimate the age effect and group difference in the regression. Group differences were also tested with $t$-tests equal variance not assumed. Neither group showed an effect of age when regression analyses were conducted for each group separately. Therefore the unequal variance is unlikely to affect interpretation of the results.
} 
belonging to the WS or TD group based on the composite score alone. Examination of individual scores showed that $91 \%$ of WS showed an N2 larger than N1, whereas $85 \%$ of TD controls showed the opposite pattern. Also consistent with the extant literature, participants with WS in the present study scored in the normal range on the Benton Face Recognition Test. We reasoned that high levels of proficiency subserved by atypical patterns of brain activity reflect brain plasticity. Thus, the first goal was confirmed, that this pattern can be considered a reliable marker of brain plasticity linked to face processing in WS.

A second goal of Study 1 was to determine if ERP markers of brain plasticity for face processing change with development. We tested the hypothesis that, consistent with behavioral findings (Karmiloff-Smith et al., 2004), children and adolescents with WS may show a qualitatively different pattern than adults. The results showed that the characteristic WS pattern (i.e., smaller $\mathrm{N} 1$ than N2), was highly reliable across development and was observed in all age groups. The regression analyses did not show age-related trends for either group. Thus the results suggest that the atypical pattern of brain activity reflected in the composite for WS is apparent by age 8 years and consistent through childhood through middle age. There was one interesting developmental finding. For those WS participants misclassified as TD, the only reliable difference was that they tended to be younger than those correctly classified.

A third goal was to examine within-group variability and individual differences on the markers. Surprisingly, the WS participants showed less variability in the composite scores than did the TD controls. Although this type of finding has been reported anecdotally, to our knowledge this is the first study to quantify the restricted variability in WS.

Taken together these results suggest that the abnormal pattern of brain activity linked to face perception in WS is a fundamental property of CNS function conferred by the deleted genes.

\section{STUDY 2. DEVELOPMENTAL CHANGES IN ERP MARKERS OF LANGUAGE PROCESSING}

Preliminary ERP studies of auditory language processing in small numbers of adults with WS, revealed two abnormally large positive ERP components at 100 and $200 \mathrm{msec}$, called the P1 and P2 (Bellugi, Mills, Jernigan, Hickok, and Galaburda, 1999). Auditory words also elicited an abnormally small negative component around $150 \mathrm{msec}$ in participants with WS. The large $\mathrm{P} 1-\mathrm{P} 2$ /small N1 pattern characteristic of WS participants was not observed in other atypical populations or in typically developing children. In general the P1/N1/P2 components are thought to be generated in different areas within primary auditory cortex including Heschel's Gyrus (Knight, Hillyard, Woods, \& Neville, 1980; Pantev, Hoke, Lehnertz, Fahrendorf, \& Stober, 1990). The amplitudes of these components are modulated by attentional processes and the physical characteristics of the stimuli (such as intensity). Early studies with small numbers of WS participants (Neville et al.,1989) raised the working hypothesis that this pattern represented abnormal auditory processing in WS indexing hyperexcitability of the auditory system and may in part be linked with the relatively good language skills in adults with WS. To test this hypothesis with larger numbers of individuals with WS it is important to study the development of these components in both typically developing children and children with WS. Therefore, in Study 2, ERPs were recorded while participants listened to words to examine the development of language-relevant brain systems in adults and children (8 to 51 years) with WS. 


\section{METHODS: AUDITORY LANGUAGE}

\section{Participants}

Study 2 included 135 TD controls and 74 individuals with WS participating in the auditory language paradigm (see Table 1). The grouping procedures were the same as in Study 1.

\section{Stimuli and Procedure}

The present study used the same stimuli and design as Holcomb et al. (1992) with TD children, and Neville et al. (1989) with adults with WS. Participants listened to a series of 80 naturally spoken sentences presented over headphones at a rate of one word every $700 \mathrm{msec}$ (Holcomb et al.,1992). Half of the sentences ended with an anomalous last word (e.g., "I have five fingers on my moon.") and half with a word judged to be the best completion (e.g., "I have five fingers on my hand"). The participant's task was to indicate whether the last word did or did not make sense with the rest of the sentence. ERPs were recorded and averaged according to grammatical and semantic categories. The ERPs analyzed here were to all of the open class words in the sentences except for the first and last words. Findings from the sentence endings including accuracy data and ERPs to the final target words are reported elsewhere for adults with WS (Fishman et al. 2011a,b). To reduce eye movements toward the response box, verbal responses to the sentence endings were used for a portion of the children with WS.

\section{Measurement of ERP Components}

The time windows for each component were set according to the criteria used in previous studies using the same stimuli with WS (Bellugi et al., 1999; Neville et al., 1989) and TD children (Holcomb et al., 1992). Due to marked decreases in peak latencies observed with increasing age for the auditory stimuli, it was necessary to use separate time windows for the different age groups. The time windows chosen were consistent with previous studies using the same stimuli (Holcomb et al., 1992). The first positive peak, P1, was defined as the most positive deflection between 10 and $112 \mathrm{msec}$ for all age groups. The first negative peak, N1, was defined as the most negative peak between 50 and $175 \mathrm{msec}$ for the adult groups, between 50 and $225 \mathrm{msec}$ for the children $8-15$ years of age. The second positive peak, P2, was defined as the most positive peak between 125 and $300 \mathrm{msec}$ for the adult groups and 125 to $350 \mathrm{msec}$ for the children between $8-15$ years of age.

\section{Composite Score for Language Processing}

To best characterize the relative amplitudes of the P1/N1/P2 components for each age group and population, composite variables were calculated by adding the peak amplitude of the $\mathrm{N} 1$ and the average amplitudes of the P1 and P2. Visual inspection of topographical maps, individual averages, and preliminary statistical analyses examining frontal, temporal, parietal, and occipital areas suggested that maximal differences between groups were observed over temporal regions. 
Thus, the composite scores were calculated from recordings over left and right temporal regions L/R22 and L/R41 (see Figure 2).

Unlike the face processing composite scores, log transformations to attenuate age related amplitude differences were not possible for the language composite scores because log transformations are not valid for negative numbers. A significant portion of the language composite scores consisted of negative values. Taking the absolute value of the score would not have been appropriate, as the polarity of the composite score was indicative of group membership. That is, positive scores were associated with WS and negative scores were more prevalent for the TD group. Therefore increased variability and larger component amplitudes at younger ages may contribute to age related trends, which were not observed in Study 1.

\section{Results}

The ERPs to auditory sentences are shown for all age groups in Figure 4 (right side), the composite scores for each participant are shown in the scatter plots on Figure 4 (left side). The composite scores reflect differences in the amplitudes of the N1 relative to the average of the two positive components P1 and P2. A composite score equal to zero reflects an N1 amplitude that is equivalent in absolute value to the size of the average of the P1 and P2 amplitudes.

The characteristic neural marker for language processing in WS consisted of a small N1 with large P1 and P2. Thus the typical WS composite score was larger (i.e., more positive than zero). Inspection of individual composite scores showed that among the participants with WS 65/74 (88\%) showed this pattern. The typical TD pattern showed an N1 that was equal to or larger than the mean amplitude of the P1 and P2. Composite scores for the TD group tended to be equal to or less than (i.e., more negative than zero). For TD participants 77/135 (57\%) showed this pattern.

Gender and IQ as potential covariates. Potential gender effects on composite scores and ERP components were examined by conducting group $\mathrm{x}$ gender ANOVAs with age as a covariate. Separate ANOVAs were conducted to examine gender effects for the individual N1, P1, and $\mathrm{P} 2$ components as well. For the language composite score, there was a significant gender $\mathrm{x}$ group interaction, $F(1,204)=4.248, p=.041$, non-significant main effect $F(1,204)=.940, p=.334$. Examination of the interaction revealed a significant gender effect within the TD group only. For the TD group, males displayed a larger negativity in the composite score than females, TD group effect of gender, $F(1,132)=8.76, p=.004$, Males mean composite $=-1.12 \mu \mathrm{v}$, Females mean $=.629 \mu \mathrm{v}$.

Analyses for the individual components showed that the gender effect was significant only for the N1. For the TD group only, males showed a larger N1 (mean $=-2.48 \mu \mathrm{v}$ ) than females (mean $=1.33)$; N1 TD group effect of gender, $F(1,132)=11.04, p=.001$, WS group $F(171)=1.656$, $p=.202$. No other main effects or interactions with gender were significant. Thus to control for possible confounds related to gender differences in the TD group, gender was factored in as a covariate in subsequent analyses.

IQ scores were only available for 63 participants in the WS group (mean VIQ $=69, S D=10$; $\mathrm{PIQ}=63, S D=11$; FSIQ $=64, S D=11$ ). The relation between Full Scale IQ (FSIQ) and ERPs was examined for each of the components. Partial correlations were conducted with age removed as a factor. There were no significant correlations with FSIQ for the composite score $r=.031$, $p=.814$; N1 $r=.054, p=-.677$; $11 r=-.142, p=.702$; nor the $\mathrm{P} 2 r=-.055$. 
Group differences. A discriminant analyses revealed that based on the language composite score alone $78 \%$ of cases across all ages were correctly classified, $\chi^{2}(1)=52.17, p=.000$, Wilks' Lambda $=.777$, Wilks' Lambda $=.636$, Eigenvalue $=2.87$. Classification results for each participant group showed that $83.7 \%$ of TD and $67.6 \%$ of WS were correctly classified.

A second discriminant analysis was conducted to examine how the composite score compared to predictions based on entering the amplitudes of the N1, P1, P2 as separate factors. Independent variables were entered together rather than in a stepwise manner. All three components significantly contributed to the discriminant function, each at $p=.000$. The analysis using these three factors correctly identified $73.7 \%$ of cases, $\chi^{2}(3)=67.973, p=.000$, Wilks' Lambda $=.718$, Eigenvalue $=3.92$. The classification results showed that TD group was correctly classified at $78.5 \%$, and WS at $64.9 \%$.

Discriminant analyses for each component separately showed correct classification at above chance levels (all $p<.0001)$, N1 (74.6\%), P1 (71.8\%), P2 (74.2\%), but all were below the composite score rate. Thus, using the composite score revealed the same or higher percent correct classification for each group than did the analysis using the three components combined, or as separate factors.

For the WS participants who were misclassified as TD, 20/24 (83\%) were adults, 12 were female. Although there were no correlations with IQ for the composite score or any single component (see above), the WS misclassified as TD had a lower FSIQ (58) than those correctly identified as WS (65), $t(60)=-2.261, p=.034$. That is, a higher IQ was more consistent with the WS ERP pattern.

Group differences across age. Of particular interest was whether group differences in the language composite were apparent across development. $T$-tests between groups at each age group showed that differences in language composite scores for WS and TD were significant at all age groups: at $8-12$ years, $t(55)=-3.764, p=.001 ; 13-17$ years, $t(49)=-5.858, p \leq .0001$; $18-29$ years, $t(70)=-5.917, p \leq .0001 ; 30-51$ years, $t(28)=-3.439, p=.001$.

To further examine age-related trends, a regression analysis was conducted using group, age, and age $\mathrm{x}$ group as factors. The regression on the language composite was significant accounting for $31 \%$ of the variance, $\mathrm{R}^{2}=.313, F(4,204)=25.477, p<.0001$. The overall effect of group was significant, group, $\mathrm{b}=.562, t(209)=9.435, p \leq .0001$. The effect of age was not significant, $t(209)=1.446, p=.150$. However, there was an interaction with age and group, $\mathrm{b}=$ $-.481, t(209)=-3.799, p=.0001$. Examination of the group $\mathrm{x}$ age interaction showed that the effect of age was significant for the WS group, age, $t(73)=-3.326, p=.001$, but did not reach significant levels for the TD controls, $t(134)=-1.707, p=.090$. For participants with WS the composite score was increasingly less positive with increasing age (i.e., more similar to the TD group).

To chart age-related changes for each of the components, regression analyses using age as the independent variable were conducted for the P1 - N1- and P2 amplitudes separately for WS and TD participants. These analyses revealed that the P1 amplitude decreased with increasing age for both the TD $t(134)=-4.492, p<.0001$ and WS $t(73)=-3.205, p<.002$ participants. The $\mathrm{N} 1$ did not change with age for the TD group, $t(134)=-1.374, p=.17$, but increased in amplitude with increasing age for the WS group, $t(75)=-3.217, p=.002$. Similarly, the P2 amplitude did not change with increasing age for the TD group, $t(134)=.518, p=.605$, but decreased with increasing age for the WS group, $t(75)=-2.664, p=.010$. 


\section{Individual Variability}

As in Study 1, we compared the range, standard deviations, and variance statistics between the TD and WS groups. In contrast to results from the faces composite, the WS group displayed similar variability on these measures as did the TD group (Table $2 b$ ). Levine's test showed that the groups did not differ in variance for the language composite, $F(3,205)=1.312, p=.272$.

\section{Correlation of Face and Language Markers}

To examine the specificity of the ERP markers for faces versus language processing, we conducted a correlation between the $z$-scores for each ERP marker. There were 56 participants who were tested on both faces and language processing in the same age range. The correlation between the markers for face and language processing was not significant, $r=.070, p=.607$. This shows that these markers function independently. That is, they are specific to the underlying cognitive processes of face and language processing, and do not simply index the extent to which a given individual might adhere to a general WS phenotype.

\section{Summary of ERP Markers of Auditory Language Processing}

The ERP marker for atypical auditory processing in WS (i.e., small N1 large P1/P2), was observed at all ages 8 years and older for individuals with WS. The results replicated previous findings with small numbers of adults with WS suggesting that the morphology of the early part of the waveform (i.e., first $200 \mathrm{msec}$ ), to auditory words is abnormal in children and adults with WS (Bellugi et al., 2000).

Unlike the marker for face processing, there were developmental trends for the WS group. In WS but not in TD controls, the relative amplitudes of the N1 to P1/P2 changed with increasing age. Analyses of the separate components suggest these age-related changes may be due to an extended maturation of the N1 and P2 for WS participants. Previous research with typically developing children and adults suggest that the amplitude of the P1 decreases with age until adolescence or adulthood, whereas the N1 and P2 increase in age until around 10 to 12 years of age (Gilley, Sharma, Dorman, \& Martin, 2005; Ponton, Eggermont, Kwong, \& Don, 2000; Wunderlich, Cone-Wesson, \& Shepherd, 2006). For the TD participants in the current study, N1 and P2 amplitudes didn't show a significant change between 8-12 years and adults, whereas the P1 amplitudes reached adult levels slightly later (i.e., at 13-15 years of age). In contrast, the WS participants showed a slightly later time course and atypical pattern of maturation. In WS participants, similar to TD controls, the P1 decreased with increasing age until 13-15 years of age. In contrast the $\mathrm{N} 1$ and $\mathrm{P} 2$ showed later development than the P1 in the WS participants, reaching adult levels at 13-15 years. Similar to previous reports of TD populations, for the WS groups the N1 increased in amplitude with increasing age. However, the P2 decreased with increasing age for WS, which is the opposite pattern from normal development suggested by earlier studies. These findings suggest there may be an extended window of maturation in WS for brain areas contributing to the N1. Longer developmental trajectories are associated with both increased vulnerability and potential for enhancement. Thus, these findings are to some extent in support of the earlier hypothesis that atypical patterns of brain activity in the auditory cortex may be related to relative proficiencies in language processing. 


\section{STUDY 3. ERP MARKERS FOR TWO ADULTS WITH PARTIAL DELETIONS FOR WS}

A growing number of studies have begun to investigate the role of specific genes, such as LIMkinase, FKBP6, and GTF2I/GTF2IRD1, by studying rare individuals with partial deletions for WS (Antonel et al., 2010; Dai et al., 2009; Karmiloff-Smith et al., 2001, 2003, 2012; Tassabehji et al.,1999). Studying individuals with typical WS deletions allows us to examine abnormalities in neurocognitive profiles related to the deletion of all of the WS region genes. In order to understand the contribution of specific WS region genes to the ERP variability, it was necessary to identify and study rare individuals with smaller or partial deletions. These provide a unique opportunity to highlight the functional significance of spared gene(s) within this region.

To begin to identify the genes contributing to the distinctive neural markers for face and language processing in WS we investigated these in two unrelated adult men with de novo, overlapping partial deletions that spared different subsets of genes located at opposite ends of the typical WS deletion (Korenberg and coworkers, in preparation). The phenotypic map of the full WS deletion as well as breakpoints for the two individuals with partial deletions is shown in Figure 5. ERP composite scores (and reported $Z$ scores and percentile rankings) for participants 5623 and 5657 are compared to those obtained from the age and gender matched normal adult controls (see Figures 6 and 7).

\section{FACE PROCESSING}

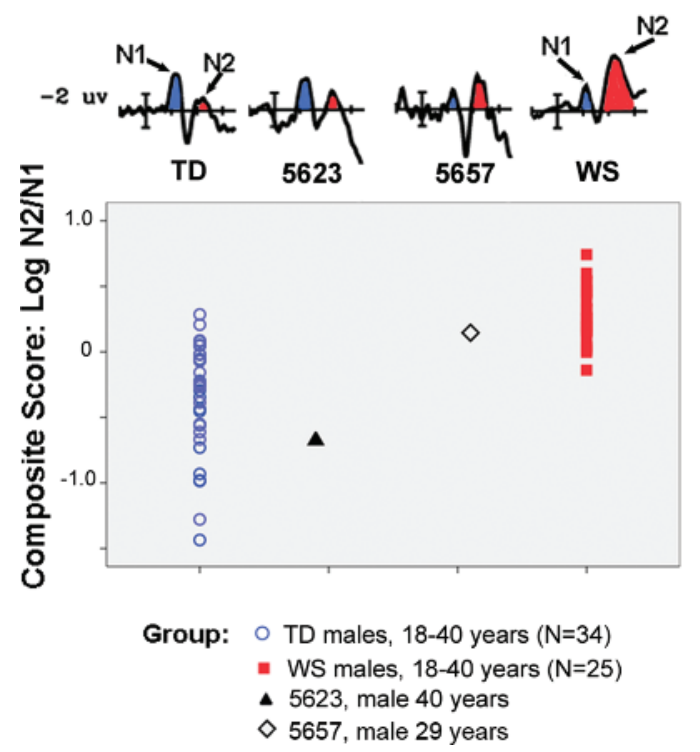

FIGURE 5 Williams Syndrome (WS) region gene map and deletion summary of atypical cases (\#5623, \#5657) and age-, gender-matched typical WS and corresponding mapping of auditory features and face event-related potential (ERP). (color figure available online) 


\section{AUDITORY LANGUAGE}

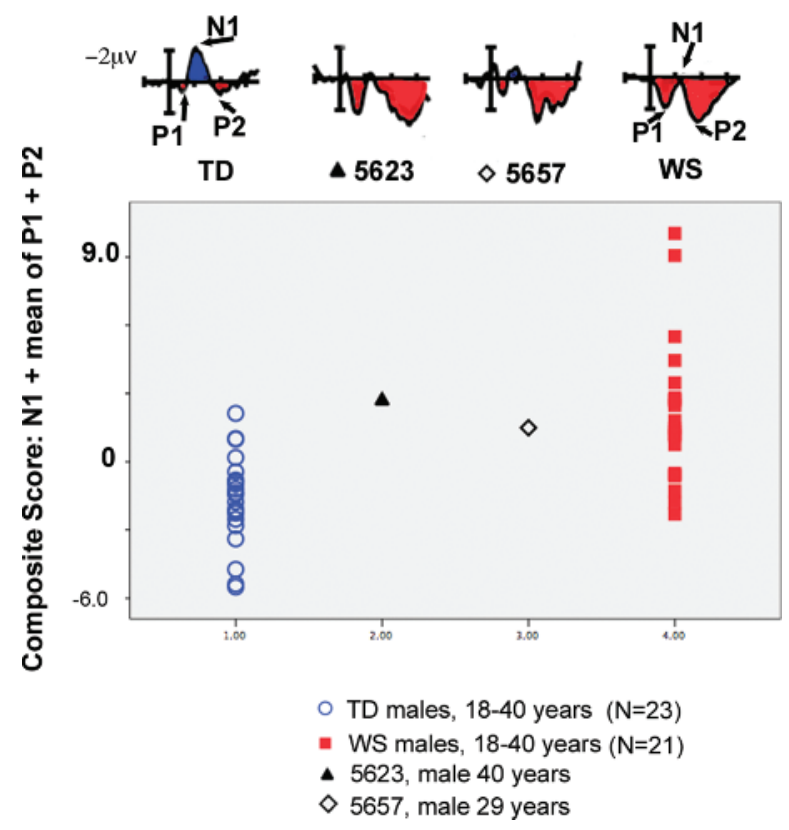

FIGURE 6 Event-related potentials (ERPs) and composite scores for face processing for two adult men with atypical deletions for Williams Syndrome (WS) and their age and gender matched controls. Participant 5623 falls outside the distribution displayed by adult males with WS and within the middle of the range for typically developing (TD) controls. In contrast, Participant 5657 falls within the range of scores for WS and at the tail of the distribution for TD controls. (color figure available online)

\section{Method and Results for Genetic Deletion Size}

High resolution FISH and then quantitative PCR were used to define the breakpoint of deleted 620 regions of participant 5623 (Korenberg et al., 1995). First, four bacterial artificial chromosomes (BACs) probes (815K3 for CALN1; 1008H17 for FKBP6, FZD9; 592D8 for ELN, LIMK1; and 1184P14 for GTF2I) marking the ends, internal, and flanking duplicated regions of the common deletion were employed as a screen for the common deletion (Korenberg et al., 2000). For participant 5623, further FISH analyses used a total of 21 sequenced linked DNAs isolated from either BACs (Korenberg et al., 2000) or cosmids (Osborne et al., 1999b) to cover the region defining the deletion breakpoints. For each FISH experiment, a total of 25 metaphase cells were evaluated and a probe mapping to chromosome $7 \mathrm{p}$ or $7 \mathrm{q}$ (unrelated regions) and labeled with a second fluorescent tag was included as a control. Five to ten images were captured using a BDS image system equipped with a CCD camera (CHD250) and stored for further analysis. The quantitative PCR was developed and used to examine the deletion region of 5623. The DNA of 5623 were isolated 


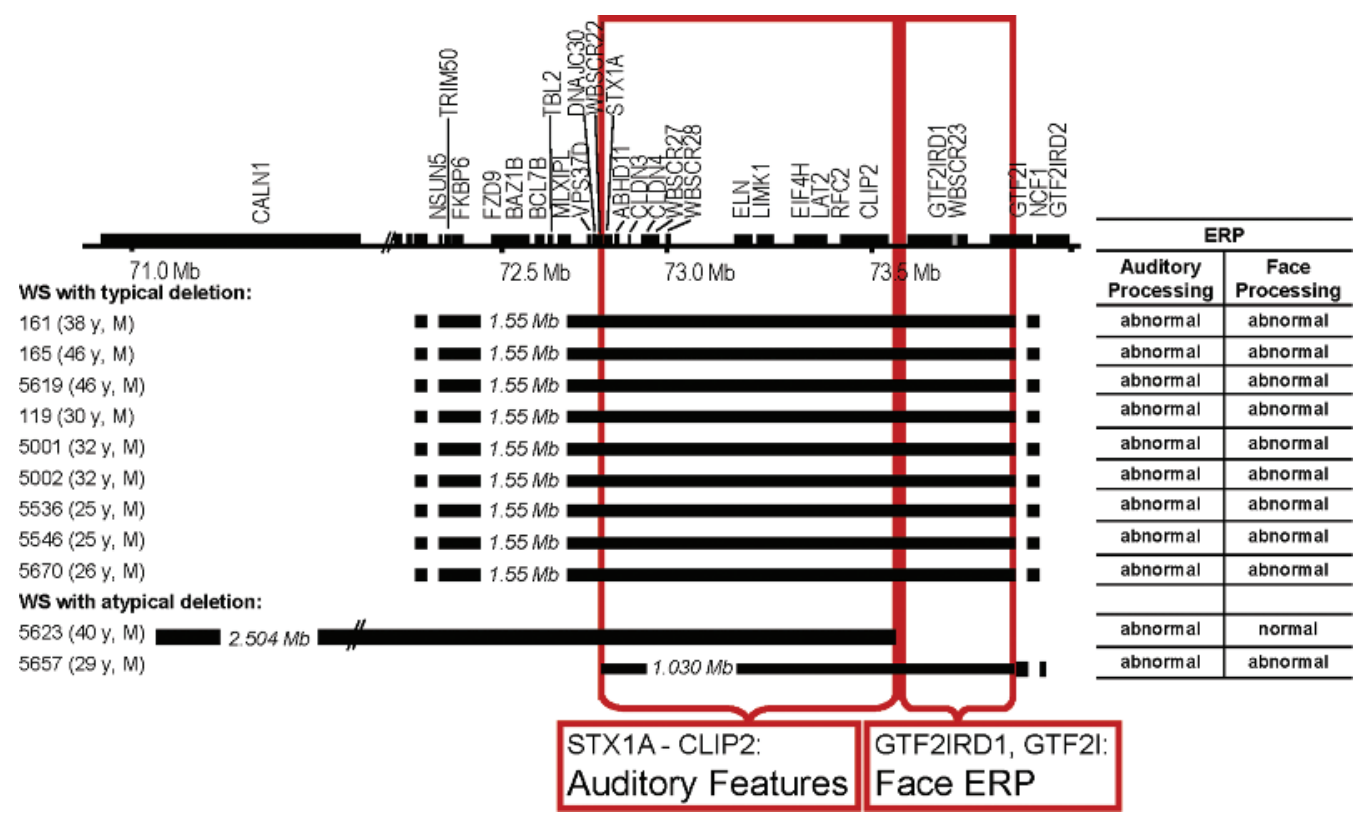

FIGURE 7 Event-related potentials (ERPs) and composite scores for auditory language processing for two adult men with atypical deletions for Williams Syndrome (WS) and their age- and gender-matched controls. Both participant 5623 and 5657 fall within the middle of the distribution for WS and at the tail end of the distribution for typically developing (TD) controls. (color figure available online)

from lymphoblast cell lines. The Taqman expression assay probes detecting the WS region genes were designed and synthesized with sequences shown in the Appendix. RNase P (VIC) was used as control. The Quantitative PCR was performed on ABI PRISM 7900HT system and the results were analyzed by using SDS 3.2.

\section{Participants}

Participant 5623 was 41 years old at the time of testing. He displayed many of the cognitive and physical features consistent with WS, including supravalvular aortic stenosis (SVAS), facial features, hyperacusis, and anxiety. Unlike typical WS he was of normal height, weight and head circumference. On cognitive tests, participant 5623 scored significantly higher on IQ tests relative to other gender and age matched participants with WS, particularly with respect to visual spatial processing. His verbal and performance IQ scores (WAIS-R, VIQ = 90, PIQ = 87, FSIQ = 88) were in the low normal range, over two standard deviations above the mean for typical WS. He performed above the norm for WS on other spatial skills, well over two standard deviations above WS means. He completed nursing school, was married and employed. Unlike typical WS, his head circumference was in the normal range, that is, he did not show the smaller posterior 
TABLE 3

Deletion Breakpoint Analysis of Participants 5623 and 5657 With Fluorescence in Situ Hybridization (FISH) and Quantitative Polymerase Chain Reaction (qPCR)

\begin{tabular}{|c|c|c|c|c|c|}
\hline \multicolumn{3}{|c|}{ FISH } & \multicolumn{3}{|c|}{$q P C R$} \\
\hline BAC/Cosmid & 5623 & 5657 & Gene & 5623 & 5657 \\
\hline $815 \mathrm{~K} 3$ & - & + & CALN1(1) & + & + \\
\hline $1008 \mathrm{H} 17$ & - & + & CALN1(2) & - & + \\
\hline $129 \mathrm{~F} 5$ & - & + & NSUN5 & - & + \\
\hline $315 \mathrm{H} 11$ & - & + & TRIM50 & - & + \\
\hline 164B05 & - & - & FKBP6 & - & + \\
\hline $82 \mathrm{C} 02$ & - & - & FZD9 & - & + \\
\hline 34B3 & - & - & BAZ1B & - & + \\
\hline 592D8 & - & - & BCL7B & - & + \\
\hline $152 \mathrm{~A} 8$ & - & - & WBSCR22 & - & + \\
\hline $102 \mathrm{~F} 12$ & - & - & STX1A & - & - \\
\hline $135 \mathrm{~F} 3$ & - & - & ELN & - & - \\
\hline 237H1 & - & - & LIMK1 & - & - \\
\hline $63 \mathrm{~F} 7$ & - & - & EIF4H & - & - \\
\hline 82B11 & - & - & LAT2 & - & - \\
\hline $209 \mathrm{C} 11$ & - & - & CLIP2 & - & - \\
\hline 47D1 & - & - & GTF2IRD1 & + & - \\
\hline 160G4 & - & - & GTF2I & + & - \\
\hline $267 \mathrm{~N} 24$ & + & - & NCF1 & + & + \\
\hline $1184 \mathrm{P} 14$ & + & - & WBSCR16 & + & + \\
\hline $239 \mathrm{C} 10$ & + & $+/-$ & & & \\
\hline $183 \mathrm{e} 1$ & $+/-$ & $+/-$ & & & \\
\hline
\end{tabular}

cortical regions characteristic of full deletion WS (Hirota et al., 2003; Korenberg et al., 2001). The results for the genetic deletion in 5623 were atypical and indicated deletion of all genes from FKBP6 through CLIP2, in common with the typical WS deletion. However, unlike typical WS, the genes GTF2IRD and GTF2I were not deleted and the deletion appeared to include a part of the gene CALN1 (Table 3 and Figure 5). Anatomical and additional standardized measures for 5623 and 5657 are available in Hoeft et al. (2013).

Participant 5657 was 29 years old at the time of testing. Like participant 5623, he displayed many of the physical and cognitive features of typical WS, including SVAS, facial features consistent with WS, hyperacusis, and anxiety. His verbal and performance IQ scores were higher (WAIS-R, VIQ $=84$, PIQ $=78$, FSIQ = 79) than is typical with WS. He had consistent employment and traveled extensively without assistance. The FISH analyses of 5657 were atypical, and indicated deletion of WS region BACs comprising genes from STX1A through GTF2I, with preservation of the flanking duplicated sequences. However, unlike typical WS, the genomic centromeric regions were not deleted, preserving (NSUN5, TRIM50, FKBP6, FZD9, BAZ1B, BCL7B, TBL2, MLXIPL, VPS27D, DNAJC30, and WBSCR22) (Table 3 and Figure 5). (For details, see Korenberg and coworkers, in preparation.) In summary, participant 5657 carried a unique and smaller deletion that included the genes encoded by GTF2iRD1 and GTF2I, a region that was not deleted in 5623, providing the opportunity to test the contribution of these genes to the abnormal neural ERP signatures of WS face processing and auditory words. 
All TD and WS male participants between 25 and 50 years from Studies 1 and 2 were selected for comparison with 5623 and 5657. The number of TD male controls for Faces $=20$ and Language $=20$, and for male adults with WS, Faces $=26$ and Language $=25$. Groups did not differ for age for faces $F(1,45)=1.667, p=.19$, or language, $F(1,44)=1.117, p=.353$.

\section{Results and Discussion of ERP Markers for Partial Deletion Cases}

Composite scores for the face and language processing paradigms were calculated for participants 5623 and 5657 and compared to age and gender matched WS and TD controls. The goal was to test the extent to which the composite scores for 5623 and 5627 on each task fell within the range for typical WS or TD participants using $z$-scores. Two $z$-scores were calculated for each composite score for faces and language paradigms: one $z$-score in comparison to the TD and one in comparison to the WS group, for each measure. A threshold of $z>2.0$ or $z<-2.0$, that is, outside the 5th to 95 th percentiles $p=.05$, was used to determine if the composite score was outside a given distribution. In this way, the $z$-scores allowed us to determine if the composite was a better fit with the WS or TD distribution. Percentile rankings for each distribution were reported when the $z$-scores were within both the WS and TD distributions.

ERPs of 5623 and 5657 to faces. ERPs to faces obtained from participant 5623 showed an ERP marker outside the distribution for men in his age range with WS, but consistent with the TD population. That is, he showed a large N1 and small N2 (Figure 6). No other individual with a typical deletion for WS has shown an ERP pattern so far outside the WS and within the TD range ( $z$ scores WS $z=-4.60$, TD $z=-.77$; percentile rankings WS $\%=0.0$, TD $\%=22$ ). Because the ERP marker for face processing in 5623 was not like WS but rather more like TD controls, this suggests that his spared genes, GTF2IRD1 and GTF2I, may be likely to contribute to the distinctive neural and structural pathways for face processing in WS. To test this gene prediction, we evaluated the ERP face-processing marker of 5657, expected to be similar to WS, predicted by his deletion of GTF2IRD1 and GTF2I.

Examination of participant 5657's ERPs to faces showed the typical WS pattern for differences between experimental conditions as well as the small N1/large N2 reflected in the composite score $(z$ scores $\mathrm{WS} z=-.61$, TD $z=1.21$; percentile rankings WS $\%=27$, TD $\%=89$ ). Thus, unlike participant 5623,5657 showed the ERP marker to faces consistent with WS, suggesting that the gene preserves (TRIM50, NSUN5, FKBP6, FZD9, BAZ1B, BCL7B, TBL2, MLXIPL, vps27D, DNAJC30, and WBSCR22) do not exert a major influence, whereas alterations in the expression of GTF2IRD1 and GTF2I do critically disturb the development and/or function of neural circuits responsible for face processing in WS.

It is important to recognize that, prior to the ERP work, face processing was thought to be "normal" and therefore, not genetically mappable in WS, based on the normal performance on the BTFR (see Karmiloff-Smith et al., 2004). The current work shows that face processing does differ in WS and is measureable by ERPs but not by cognitive testing (i.e., BTFR). This difference illustrates that genetic dissection of behavior requires the identification of "endophenotypes," neural measures that capture and distinguish genetically defined human subsets and thereby, the neural substrates of human cognition.

ERPs of 5623 and 5657 for auditory language. Unlike the composite for face processing, the composite score to auditory language processing for 5623 was within the distribution for WS 
(WS $z=.24$ ) and outside the 95th percentile of the range for normal adults (TD $z=2.10$ ). The N1 and P2 to auditory stimuli are generated in different fields of Heschel's gyrus in the superior temporal lobe (Hari et al, 1987; Knight et al., 1980; Pantev et al., 1980), a region that is larger in WS and could be associated with their proficiencies in language. Indeed, 5623 displayed the auditory ERP composite score at the WS end of the range and far outside the TD range. Anatomical measures for the superior temporal gyrus (STG) for 5623 are characteristic of WS (Hoeft et al., 2013). This suggests that in contrast to the genetics of the face processing ERP marker, it is the genes that are deleted in 5623 (region including TRIM50 through CYLN2) that are involved in the pathways underlying the ERP paradigm for auditory words, and not GTF2IRD1 or GTF2I.

The language ERPs for participant 5657 were of particular interest in that they were WS-like for the P1-N1-P2 composite score, as seen in 5623, but were in the normal range for later components that are related to cognitive ability (detailed below). Specifically, in 5657, the composite score for auditory words fell within the typical range for WS (WS $z=-.24$, TD $z=1.41$; percentile rankings WS $\%=40$, TD $\%=92$ ), (Figures 6 and 7), while some of the later components showed ERP patterns more in line with age and gender matched TD controls.

In TD adults, ERPs to the two major word classes presented in auditory sentences (open class or content words versus closed class or grammatical function words) show distinct ERP components that differ in timing and distribution (Holcomb et al., 1992; Weber-Fox \& Neville, 1996). Specifically in TD controls, "function" words elicit a left anterior negativity, and a later posterior negativity; whereas "content" words elicit a greater N400 than do function words that are indexed independently of frequency, length, and imageability (Neville, Mills, \& Lawson, 1993). That is, for TD controls, open and closed class words are mediated by non-identical brain systems. However, in WS, most individuals do not show these ERP differences to "content" versus "function" words nor a left greater than right asymmetry to "function" words (Mills et al. 1998, 2000; Bellugi et al., 1999; St. George \& Mills, 2000).

Unlike adults and children with the typical WS deletion, participant 5657 showed ERP differences to open and closed class words that were normal in latency, polarity and lateral distribution. He also showed a left greater than right asymmetry to grammatical function words that is not typically found in the typical WS group. The more normal ERP components later in the waveform may reflect specific genetic differences in his higher cognitive (WAIS-R verbal IQ of 84) and linguistic ability as they do in TD and our data indicate that they are more variable within both the WS and control populations.

These results suggest that that the lack of distinct patterns of neural activity to content versus grammatical function words in WS. This suggests that similar brain systems are likely involved in processing both open and closed class words and may be linked to genes in the WS regions. In particular, they may implicate the genes from TRIM50 through WBSCR22 (preserved in 5657) in the response to function words. Source localization of the N400 in TD adults suggests involvement of the STG (Khateb, Pegna, Landis, Mouthon, \& Annoni, 2010). Both the STG and N400 anomaly effect are larger in WS than controls.

We offer the working hypothesis that in WS, the STG may be responsible for processing both open and closed class words. The lack of a larger N400 to content than function words, and lack of the ERP asymmetry over the left anterior region to function words seen in WS and 5623, decrease the likelihood that GTF2IRD1 and GTF2I are important for these linguistic features. However, the more typical ERP differences to open versus closed class words in TD controls, and 5657 combined with the absence in WS, may implicate a role for the genes in the region 
from TRIM50 through WBSCR22 in response to words conveying meaning and to words with grammatical function. These results are significant in suggesting links between genes and brain function indexing later cognitive components and begin to provide a basis for dissecting the genetic contributions to the development of human language.

\section{GENERAL DISCUSSION AND CONCLUSIONS}

Our findings from a large sample of individuals with the typical deletion for WS provide strong evidence that sensory and attentional mechanisms for two cognitive functions mediated by ventral stream processes, face and auditory language processing, are abnormally organized in WS across development. These findings are in marked contrast to reports that ventral stream processes are spared or normally organized in WS (Landau et al., 2006; Meyer-Linderberg, 2006). Moreover, these findings are the first to define the developmental trajectories of atypical neural activity that are associated with relative proficiencies in WS. The consistent patterns of distinct ERP markers for abnormal face and language processing in WS from age 8 through middle age show that neural mechanisms that underlie abnormal face and language processing in WS adults are established in early childhood and are powerful for dissecting genes contributing to these cognitive processes. Moreover, the lack of correlation of $z$-scores for the two markers in full deletion WS, combined with the results from participants with partial deletions, suggests that these ERP markers are not related to a single WS gene or neural process. Rather, face processing and language are affected by distinct gene subsets in the WS region and affect the development and function of different neural substrates.

The ERP markers also illustrate that face processing is abnormal in WS across development, despite their near normal range performance on standard cognitive tests. The ERPs thereby provide a brain-based endophenotype that was utilized here to identify genetic contributions for WS features in persons with smaller deletions, first showing that partial deletions can account for the characteristic WS or TD profile. Our findings establish that WS cognitive features are not due to a single gene but rather strongly support the involvement of distinct subsets of WS genes in the specific neurocognitive features of WS (Dai et al., 2009). Similar to the shared congenital heart disease that is caused by shared deletion of the gene encoding elastin, the facial and cognitive features 5623 shares with WS such as deficits in language and ERPs to auditory words, are in part due to the deleted genes that he shares with typical WS. Furthermore, the preserved visuospatial cognition observed in 5623 confirms earlier work (Hirota, 2003), and does not appear to be the effect of a dose-dependent relationship between the size of the deletion and cognition, as participant 5657 possesses a smaller-sized deletion, but in a different locus, yet did not demonstrate a concomitant preservation in visuospatial task performance.

It is of interest that the ERP data demonstrated a consistent pattern of early neural activity in response to auditory language processing in WS and both partial deletion cases, suggesting the involvement of genes deleted in common (STX1A throughCYLN2). The neuroanatomic origin of this activity is unclear. In contrast, the late waveforms of 5657 are within the normal range in the language paradigm and may be related to his preserved genes. This hypothesis is supported in part by the observation that participant 5657's performance on many language measures was better than both the full deletion WS group and 5623. That is, the spared genes in 5657 may have some neurological significance with respect to development of cerebral structures or connectivity 
patterns supporting language. Moreover, the difference in the early versus late ERP waveforms suggests that the genes and neural structures subserving these different aspects of language, differ, with those genes that are preserved, being more involved in ERP responses to closed (function) and open (content) class words, and possibly related to the disrupted hemispheric asymmetry seen in WS. Given the modest magnitude of the increased language scores obtained from 5657, further study is clearly needed.

The current study is not without limitations. The neural markers provide a first step to generate hypotheses regarding gene brain relations across development. In future studies, the functional significance of the neural markers for face and language processing should be established by correlating performance on standardized and experimental behavioral tests on a variety of different cognitive measures. A second limitation was that there were only two participants with partial deletions. It is critical to continue to test new partial deletion cases on these markers to strengthen gene/brain associations and test the hypotheses generated here. Replication of the study with other individuals with similar partial deletions would strengthen the generalizability of the results. It will also be important to study children with partial deletions to examine how the spared genes interact with changes in brain and cognitive development.

In conclusion, we propose that there may be at least two gene groups that affect language and at least one affecting face processing brain activity in WS: (1) STX1A through CYLN2, including LIMK1, and others, are candidates for a role in the deleterious effects of gene deletion on part of WS' language functions including the early ERP-related verbal performance measured here. (2) TRIM50 through WBSCR22, including FZD9, WSTF, BAZ1B, TBL2, WBSCR28, CLDN3, are candidates for a role in the later ERP waveforms related to closed and open class words in WS. (3) For brain activity related to face processing, we propose that the altered expression of genes encoding GTF2IRD1 and GTF2I affects the development and adult function of brain substrates related to visual-spatial functions including those decreased in WS, the posterior parietal and occipital cortices. Future studies of high temporal resolution brain activity employing ERPs, particularly those studied in large cohorts that are characterized over development, will provide a unique opportunity to refine the genetic and epigenetic contributions to cognition, behavior, and their neural substrates using partial deletions combined with next generation sequencing and novel approaches to tissue specific gene expression.

\section{ACKNOWLEDGMENTS}

We thank all the participants and their families who so generously gave their time to take part in this study. We thank Terra Llamas, Chantel Prat, and Marie St. George for help in collection and processing of the data. The cosmids were generous gifts from Dr. L.C. Tsui at The Hospital for Sick Children, Toronto, Ontario, Canada.

\section{REFERENCES}

Annaz, D., Karmiloff-Smith, A., Johnson, M. H., \& Thomas, M. S. C. (2009). A cross-syndrome study of the development of holistic face recognition in children with autism, Down syndrome, and Williams syndrome. Journal of Experimental Child Psychology, 102, 456-486.

Atkinson, J., Braddick, O., Rose, F. E., Searcy, Y. M., Wattam-Bell, J., \& Bellugi, U. (2006). Dorsal-stream motion processing deficits persist into adulthood in Williams syndrome. Neuropsychologia, 44, 828-833. 
Bellugi, U., \& St. George, M. (2001). Journey from cognition to brain to gene: Perspectives from Williams Syndrome. London, England: MIT Press.

Bellugi, U., Lichtenberger, L., Jones, W., Lai, Z., \& St. George, M. (2000). The neurocognitive profile of Williams Syndrome: A complex pattern of strengths and weaknesses. Journal of Cognitive Neuroscience, 12, (Suppl. 1), 7-29.

Bellugi, U., Lichtenberger, L., Mills, D., Galaburda, A., \& Korenberg, J. (1999b). Bridging cognition, brain and molecular genetics: Evidence from Williams syndrome. Trends in Neuroscience, 22, 193-236.

Benton, A., Hamsher, K., Varney, N. R., \& Spreen, O. (1983). Benton Test of Facial Recognition. New York, NY: Oxford University Press.

Eckert, M. A., Hu, D., Eleiz, S., Bellugi, U., Galaburda, A., Korenberg, J., . . Reiss, A. (2005). Evidence for superior parietal impairment in Williams syndrome. Neurology, 64, 152-153.

Fishman, I., Yam, A., Bellugi, U., \& Mills, D. (2011b). Language and sociability: Insights from Williams Syndrome. Journal of Neurodevelopmental Disorders, 3(3), 185-192.

Fishman, I., Yam, A., Bellugi, U., Lincoln, A., \& Mills, D. (2011a). Contrasting patterns of language-associated brain activity in Autism and Williams Syndrome. Social Cognitive and Affective Neuroscience, 6(5), 630-638.

Galaburda, A. M., \& Bellugi, U. (2000). Multi-level analysis of cortical neuroanatomy in Williams syndrome. Journal of Cognitive Neuroscience, 12(Suppl. 1), 74-88.

Galaburda, A. M., Holinger, D. P., Bellugi, U., \& Sherman, G. F. (2002). Williams syndrome: Neuronal size and neuronalpacking density in primary visual cortex. Archives of Neurology, 59, 1461-1467.

Gilley, P. M., Sharma, A., Dorman, M., \& Martin, K. (2005). Developmental changes in refractoriness of the cortical auditory evoked potential. Clinical Neurophysiology, 116, 648-657.

Grice, S. J., de Haan, M., Halit, H., Johnson, M. H., Csibra, G., Grant, J., \& Karmiloff- Smith, A. (2003). ERP abnormalities of visual perception in Williams syndrome. NeuroReport, 14, 1773-1777.

Gothelf, D., Farber, N., Raveh, E., Apter, A., \& Attias, J. (2006). Hyperacusis in Williams syndrome: Characteristics and associated neuroaudiologic abnormalities. Neurology, 66, 390-395.

Haas, B., Mills, D. L., Yam, A., Hoeft, F., Korenberg, J., Bellugi, U. \& Reiss, A. (2009). Genetic influences on sociability: Heightened amygdala reactivity and event-related responses to positive social stimuli in Williams Syndrome. Journal of Neuroscience, 29(4), 1132-1139.

Hari, R., Pelizzone, M., Makela, J. P., Hallstrom, J., Leinone, L., \& Lounasmaa, O. V. (1987). Neuromagnetic responses of the human auditory cortex to on- and offsets of noise bursts. Audiology, 26, 31-43.

Hoeft, F., Dai, L., Haas, B., Sheau, K., Mimura, M., Mills, D., . . Reiss, A. (2013, in revision). Mapping genetically controlled neural circuitries of social behavior and visuo-motor integration by examination of atypical deletions with Williams Syndrome.

Holcomb, P. J., Coffey, S. A., \& Neville, H. J. (1992). Visual and auditory sentence processing: A developmental analysis using event-related brain potentials. Developmental Neuropsychology, 8, 203-241.

Holinger, D. P., Bellugi, U., Mills, D. L., Korenberg, J. R., Reiss, A. L., . . Galaburda, A. M. (2005). Relative sparing of primary auditory cortex in Williams Syndrome. Brain Research, 1037, 35-42.

Karmiloff-Smith, A., \& Thomas, M. (2003). What can developmental disorders tell us about the neurocomputational constraints that shape development? The case of Williams syndrome. Developmental Psychopathology, 15, 969-990.

Karmiloff-Smith, A., Scerif, G., \& Thomas, M. (2001). Different approaches to relating genotype to phenotype in developmental disorders. Developmental Psychobiology, 40, 311-322. doi:10.1002/dev.10035

Karmiloff-Smith, A., Thomas, M., Annaz, D., Humphreys, K., Ewing, S., Brace, N., . . . Campbell, R. (2004). Exploring the Williams Syndrome face-processing debate: The importance of building developmental trajectories. Journal of Child Psychology \& Psychiatry, 45, 1258-1274.

Karmiloff-Smith, A., Broadbent, H., Farran E. K., Longhi, E., D’Souza, D., Metcalfe, K., ... Sansbury, F. (2012). Social cognition in Williams Syndrome: Genotype/phenotype insights from partial deletion patients. Frontiers in Psychology, 3 .

Kaur, M., Lagopoulos, J., Ward, P. B., Watson, T. L., Naismith, S. L., Hickie, I. B., \& Hermens (2012). Mismatch negativity/P3a complex in young people with psychiatric disorders: A cluster analysis. PLOS One, 12, e51871.

Khateb, A., Pegna, A. J., Landis, T., Mouthon, M. S., Annoni, J. M. (2010). On the origin of the N400 effects: An ERP waveform and source localization analysis in three matching tasks. Brain Topography, 23(3), 311-320.

Kippenhan, J. S., Olsen, R. K., Mervis, C. B., Morris, C. A., Kohn, P., Meyer-Lindenberg, A., \& Berman, K. F. (2005). Genetic contributions to human gyrification: Sulcal morphometry in Williams Syndrome. Journal of Neuroscience, 25, 7840-7846. 
Klein, C., Wendling, K., Huettner, P., Ruder, H., \& Peper, M. (2006). Intra-subject variability in attention-deficit hyperactivity disorder. Biological Psychiatry, 60, 1088-1097.

Knight, R., Hillyard, S. A., Woods, D. L., \& Neville, H. (1980). The effects of frontal and temporal-parietal lesions on the auditory evoked response in man. Electroencephalography and Clinical Neurophysiology, 50, 112-124.

Korenberg, J. (1995). Mental modeling. Nature Genetics, 11(2), 109-111.

Korenberg, J. R., Bellugi, U., Salandanan, L. S., Mills, D. L., \& Reiss, A. L. (2003). Williams Syndrome: A neurogenetic model of human behavior. Nature Encyclopedia of the Human Genome (pp 757-766). Hampshire, England: Macmillan, Nature Publishing Group.

Landau, B., Hoffman, J. E., \& Kurz, N. (2006). Object recognition with severe spatial deficits in Williams Syndrome: Sparing and breakdown. Cognition, 10, 483-510.

Leonard, H. C., Annaz, D., Karmiloff-Smith, A., \& Johnson, M. H. (2011). Brief report: Developing spatial frequency biases for face recognition in Autism and Williams Syndrome. Journal of Autism Developmental Disorders, 41, 968-973. doi:10.1007/s10803-010-1115-7

Levitin, D. J., Menon, V., Schmitt, J. E., Eliez, S., White, C. D., Glover, G. H., . . Reiss, A. L. (2003). Neural correlates of auditory perception in Williams Syndrome: An fMRI study. NeuroImage, 18, 74-82.

Levitin, D. J., Cole, K., Chiles, M., Lai, Z., Lincoln, A., \& Bellugi, U. (2005). Characterizing the musical phenotype in individuals with Williams Syndrome: Erratum. Child Neuropsychology, 11, 477.

Loo, S. K., \& Makeig, S. (2012). Clinical utility of EEG in attention deficit/hyperactivity disorder: A research update, Neurotherapeutics 9(3), 569-587. doi:10.1007/s13311

Luck, S. J. (2005). An introduction to the event-related potential technique. Cambridge, MA: The MIT Press.

Martens, M. A., Wilson, S. J., \& Reutens, D. C. (2008). Research review: Williams Syndrome: A critical review of the cognitive, behavioral, and neuroanatomical phenotype. Journal of Child Psychology and Psychiatry, and Allied Disciplines, 49(6), 576-608.

McCleery, J.P., Allman, E., Carver, L.J., \& Dobkins, K. R. (2007).Abnormal magnocellular pathway visual processing in infants at risk for autism. Biological Psychiatry, 62, 1007-1014.

Mendes, M., Silva, F., Simoes, L., Jorge, M., Saraiva, J., \& Castelo-Brancoa, M. (2005). Visual magnocellular and structure from motion perceptual deficits in a neurodevelopmental model of dorsal stream function. Cognitive Brain Research, 25, 788-798.

Meyer-Lindenberg, A., Mervis, C. B., \& Berman, K. F. (2006). Neural mechanisms in Williams Syndrome: A unique window to genetic influences on cognition and behaviour. Nature Reviews Neuroscience, 7, 380-393.

Meyer-Lindenberg, A., Hariri, A. R., Munoz, K. E., Mervis, C. B., Mattay, V. S., Morris, C. A., \& Berman, K. F. (2005). Neural correlates of genetically abnormal social cognition in Williams syndrome. Nature Neuroscience, 8, 991-993.

Mills, D. L. (1998). Electrophysiological markers of abnormal brain function in Williams Syndrome. Cognitive Neuroscience Society, Annual Meeting. Supplement of the Journal of Cognitive Neuroscience, (Abstract), 10.

Mills, D. L., Alvarez, T. D., St. George, M., Appelbaum, L. G., Bellugi, U., \& Neville, H. (2000). Electrophysiological studies of face processing in Williams syndrome. Journal of Cognitive Neuroscience, 12(Suppl. 1), 47-64.

Mitchell, T. V., \& Neville, H. J. (2003). Effects of age and experience on development of neurocognitive systems. In A. Zani, A. M. Proverbio, \& M. I. Posner (Eds.), Cognitive electrophysiology of mind and brain (pp. 225-244). : Elsevier Academic Press.

Mobbs, D., Garrett, A. S., Menon, V., Rose, F. E., Bellugi, U., \& Reiss, A. L. (2004). Anomalous brain activation during face and gaze processing in Williams syndrome. Neurology, 62, 2070-2076.

Molfese, D. L. (2000). Predicting dyslexia at 8 years using neonatal brain responses. Brain and Language, 72, $238-245$.

Neville, H. J., Holcomb, P. J., \& Mills, D. L. (1989). Auditory sensory and language processing in Williams syndrome: An ERP study. Journal of Clinical and Experimental Neuropsychology, 11, 52.

O’Hearn, Ki., Roth, J. K., Courtney, S. M., Luna, B., Street, W., Terwillinger, R., \& Landau, B. (2011). Object recognition in Williams syndrome: Uneven ventral stream activation. Developmental Science, 14(3), 549-565.

Pantev, C., Hoke, M., Lehnertz, K., Fahrendorf, G., \& Stober, U. (1990). Identification of sources of brain neuronal activity with high spatiotemporal resolution through combination of neuromagnetic source localization (NMSL) and magnetic resonance imaging (MRI). Electroencephalography and Clinical Neurophysiology, 75, 173-

Pellicano, E., Gibson, L., Maybery, M., Durkin, K., \& Badcock, D. R. (2005). Abnormal global processing along the dorsal visual pathway in autism: A possible mechanism for weak visuospatial coherence? Neuropsychologia, 43(7), 1044-1053.

Pinker, S. (1994). The language instinct. London, England: Allen Lane. 
Ponton, C. W., Eggermont, J. J., Kwong, B., \& Don, M. (2000). Maturation of human centralauditory system activity: Evidence from multi-channel evoked potentials. Clinical Neurophysiology, 111(2), 220-236.

Pratt, H. (2012). Sensory ERP components. In S. J. Luck \& S. S. Kappenman (Eds.), The Oxford handbook of eventrelated potential components (pp. 89-114). Oxford, England: Oxford University Press.

Reiss, A. L., Eliez, S., Schmitt, J. E., Straus, E., Lai, Z., Jones, W., \& Bellugi, U. (2000). Neuroanatomy of Williams Syndrome: A high-resolution MRI study. Journal of Cognitive Neuroscience, 12(Suppl. 1), 65-73.

Smit, D. J. A., Posthuma, D. I., Boomsa, E. J. C., \& De Geus, E. J. C. (2007). Heritability of anterior and posterior visual N1. International Journal of Psychophysiology, 66, 196-204.

St. George, M., Mills, D., \& Bellugi, U. (2000). ERPs during auditory language comprehension in Williams Syndrome: The effects of word frequency, imageabilty and length on word class. Neuroimage, 11, 357.

Ullman, M. T., \& Pierpont, E. I. (2005). Specific language impairment is not specific to language: The procedural deficit hypothesis. Cortex, 41, 399-433.

Wang, P. P., Doherty, S., Rourke, S. B., \& Bellugi, U. (1995). Unique profile of visuo-spatial skills in a genetic syndrome. Brain and Cognition, 29(1), 54-65.

Weber-Fox, C. M., \& Neville, H. J. (1996). Maturational constraints on functional specializations for language processsing: ERP and behavioral evidence in bilingual speakers. Journal of Cognitive Neuroscience, 8(3), 231-256.

Wunderlich, J. L., Cone-Wesson, B. K., \& Shepherd, R. (2006). Maturation of the cortical auditory evoked potential in infants and young children. Hearing Research, 212, 185-202. 


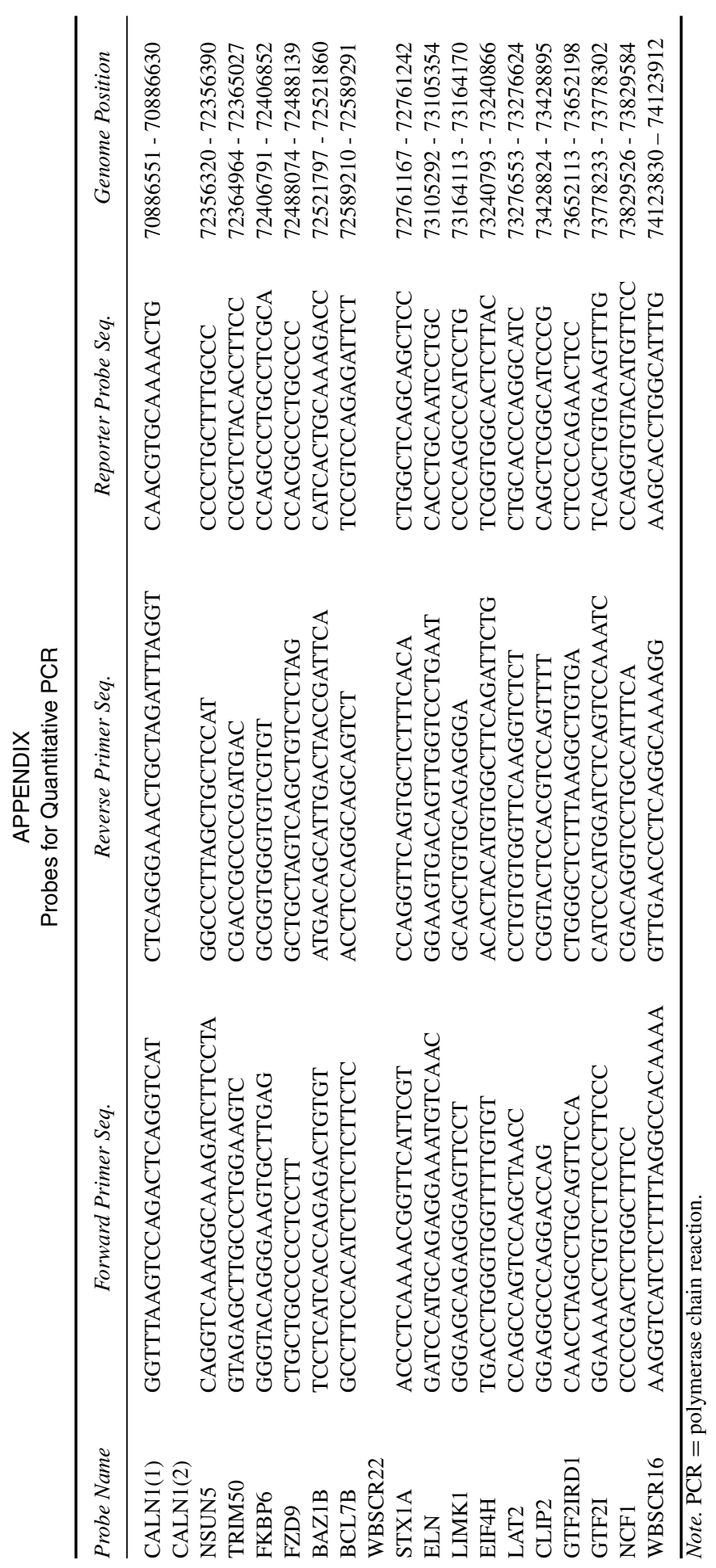

